\title{
Large-eddy simulation of separated flow over a three-dimensional axisymmetric hill
}

\author{
M. GARCÍA-VILLALBA ${ }^{1} \dagger, N$. LI I ${ }^{2}$ W. RODI \\ AND M. A. LESCHZINER ${ }^{2}$ \\ ${ }^{1}$ Institute for Hydromechanics, University of Karlsruhe, Kaiserstr 12, 76128 Karlsruhe, Germany \\ ${ }^{2}$ Aeronautics Department, Imperial College London, Prince Consort Rd, London SW7 2AZ, UK
}

(Received 2 April 2008 and in revised form 16 December 2008)

The paper examines, by means of highly resolved large-eddy simulations, the fluid mechanic behaviour of an incompressible, turbulent flow separating from an axisymmetric, hill-shaped obstacle. The hill is subjected to a turbulent flat-plate boundary layer of thickness half of the hill height, developing within a large duct in which the hill is placed. The Reynolds number based on the hill height and the free-stream velocity is 130000 , and the momentum-thickness Reynolds number of the boundary layer is 7300 . Extensive and detailed experimental data are available for these same conditions, and these are compared with the simulations. Two entirely independent simulations are reported, undertaken by different groups with different codes and different grids. They are shown to agree closely in most respects, and any differences are carefully identified and discussed. Mean-flow properties, pressure distributions and turbulence characteristics are reported and compared with the experimental data, with particular emphasis being placed on the separation region that is formed in the leeward of the hill and on the near wake. The connection between the wall topology of the mean flow and the secondary motions in the wake is discussed in detail. Finally, some aspects of the unsteady flow are analysed, including the unsteadiness of the separation process and structural features and coherent motions in the wake.

\section{Introduction}

The separation of a turbulent boundary layer from a gently curving convex surface is a process of primary concern in numerous external and internal fluids engineering components and applications, because of its major impact on operational characteristics that are linked to the pressure distribution, form and viscous drag, losses, vibrations, noise and heat transfer. Highly loaded aircraft wings, fuselages at high incidence, streamlined car bodies, low-pressure turbine blades and curved ducts are but a few examples in which the presence or absence of separation can have a decisive influence on the ability of the device in question to perform effectively and safely. It follows, therefore, that gaining insight into the underlying physical mechanisms at play, and developing an ability to represent them with a high degree of fidelity and confidence are of major interest, both from fundamental and practical perspectives. 
Although all separated flows share a number of common features, associated with the formation and, usually, reattachment of a highly turbulent separated shear layer, there are major differences between flows separating from a sharp edge and those separating from a gently curving surface. Important differences also exist between separation from geometrically two-dimensional and three-dimensional surfaces. The principal difference is that the separation location in the latter group is not fixed by the geometry, but varies substantially in both time and space, due to a sensitive two-way interaction between the separation process itself and the outer flow. In particular, the separation topology is continually modified by the large-scale vortical motions shed from the surface, and vice-versa. Additional two-way interactions arise from eddies originating from the boundary layer upstream of the separation region, passing over and around the separating flow, and from the dynamics of the reattachment process, the latter by the combined action of backward-flowing structures and the backward transmission of pressure perturbations from the impingement process at reattachment. In terms of time-averaged flow properties, the shape of the separated region has a major impact on the pressure field, which affects, in turn, the time-mean separation location. When separation is incipient or tenuous - a situation that is often of major importance as a limiting operational condition - even slight variations in geometry or boundary conditions can trigger or suppress separation. Finally, in compressible flows, another frequently encountered type of two-way interaction arises from the formation of a shock wave that causes boundary-layer separation, the spatial and temporal details of which influence, as well as being sensitive to, the unsteady and time-mean shock positions.

Despite the prevalence and importance of separation from curved surfaces, little has been published that provides satisfactory insight into the dynamics of the separation process and the extent to which these dynamics can be linked to the statistical properties of the flows in question. This applies, in particular, to three-dimensional separation, which is almost invariably characterized by a complex topology of curved separation and attachment lines, focal and saddle points, and vortices, the last realigned by the main forward motion to give rise to strong rotational secondary motion in the recovering wake.

While Reynolds-averaged Navier-Stokes (RANS) methods are, of course, routinely applied to separated flows in engineering practice, such applications are not insightful in respect of the fundamental physics at play, the level of confidence in the solutions derived from them is low, model-dependence is very high, unquantifiable and illunderstood, and the relationship between the modelling framework and physical reality is tenuous. Quite apart from not resolving unsteady features, current singlepoint RANS models are fundamentally constrained by the fact that they were designed for, and calibrated by reference to, flows in which the macro-length scale $L=k^{3 / 2} / \varepsilon$ and eddy-turnover time scale $T=k / \varepsilon$ are substantially smaller and shorter, respectively, than the corresponding mean-flow distortion scales $L_{D}=|\nabla \mathbf{U}| /|\nabla(\nabla \mathbf{U})|$ and $T_{D}=|\nabla \mathbf{U}| /(\partial|\nabla \mathbf{U}| / \partial t)$. However, separated flows are characterized by precisely the opposite relationship between corresponding scales, and the models are therefore ill-suited to such conditions, even if they resolve anisotropy and are designed to respond statistically correctly to the effects of curvature and normal straining.

Experimental studies, some noted below, provide useful information on statistical properties, but rarely illuminate the turbulence dynamics (although time-resolved Particle Image Velocimetry is promising in this respect), especially short-time-scale events, that give rise to the observed statistical behaviour. On the other hand, highly resolved simulations of separated flows can offer such insight, but are rare at 


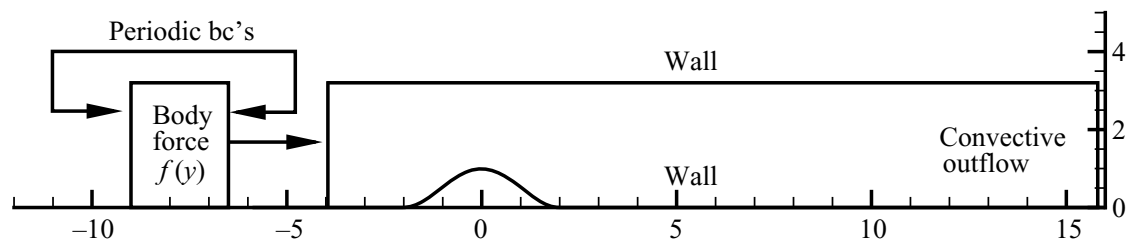

FIGURE 1. Sketch of the computational domain and boundary conditions.

elevated Reynolds numbers that are pertinent to practical applications. The review of simulations, targeting specifically separation from two-dimensional and, preferentially, three-dimensional curved surfaces, is at the centre of the remainder of this section.

One class of curved-surface configurations that has attracted significant attention and is fundamentally relevant to the present subject matter involves flows separating from sinusoidally varying surfaces, representing idealized atmospheric flows over wavy terrain, or from two-dimensional hill-shaped obstructions (undulations). A recent study by Fröhlich et al. (2005) for a streamwise-periodic train of such hills in a channel at $R e_{h}=10595$ highlights the strongly time- and space-varying separation and reattachment processes and provides a wealth of statistical and flow-structure information that explains a number of particular features in the statistical properties extracted from the simulation. For example, the high level of turbulence in the early stretch of the separated shear layer is shown to be linked not only to instability inside the shear layer, but also to the intense fluctuations in the separation location and the associated dynamics - an observation that negates the concept of the pre-separated flow being in any way akin to an attached boundary layer. Other studies illuminating related unsteady interactions are the curved-ramp flow investigated experimentally by Song, DeGraaff \& Eaton (2000) and Aubertine \& Eaton (2005), with corresponding LES studies performed by Wasistho \& Squires (2001), and a trailing-edge hydrofoil, which was the subject of an LES study by Wang \& Moin (2002), corresponding to much earlier experimental measurements by Blake (1975).

While some of the above investigations on separation from two-dimensional surfaces provide a pertinent background to the flow studied herein, especially on the unsteady nature of the separation process, three-dimensional separation is, as noted already, substantially more complex, involving highly skewed flow close to the surface, curved separation and attachment lines, various vortices deflected by or shed from the curved surface, and a highly three-dimensional wake. Such flows are, therefore, much more challenging to measure and to compute accurately, and there are very few configurations offering the opportunity to study fundamental mechanisms to any significant depth, by reference to and aided by experimental observations.

A generic configuration that combines most challenging features encountered in practical three-dimensional separated flows is around an axisymmetric threedimensional hill subjected to a turbulent boundary layer as shown in figure 1 . As in the case of flow over two-dimensional undulating surfaces, early studies on this geometry were pursued in the context of atmospheric boundary layers interacting with hills and mountains. An early seminal paper, reporting wind-tunnel and water-flume measurements for both uniform property and stratified flows, is by Hunt \& Snyder (1980). Although focusing primarily on the influence of stable stratification on the mean-flow characteristics, the paper also describes, based on the application of a range of flow-visualization techniques and hot-wire anemometry, the fundamental meanflow features in non-buoyant flow, including the interaction of the oncoming boundary 
layer with the circular hill, the topology on the hill surface and the characteristics of the separated flow. Identified, in particular, are the curved separation line in the leeward part of the surface that terminates in focal points, the outer horseshoe vortex associated with the boundary layer deflected around the hill, the recirculation zone in the wake across the centre plane of the hill, and the reattachment saddle point terminating the closed recirculation zone and marking the beginning of the recovering wake.

A more recent study by Apsley \& Castro (1997), also undertaken in the context of atmospheric flows, exemplifies the status of computational approaches applied by the mid 90 s to three-dimensional separation from hill-shaped surfaces. In that study, a $k-\varepsilon$ turbulence model was used to predict the flow and scalar dispersion in stratified flow around a real hill and its laboratory model, the focus being on qualitative flow features and ground-level concentration of the scalar tracer. The first LES of a flow over a three-dimensional hill, in the context of atmospheric flows, was performed by Ding \& Street (2003), who reported simulations with and without density stratification. However, they only studied the transient regime and did not consider the statistically steady state.

Renewed interest in analysing and predicting three-dimensional separation was provoked by a series of detailed experimental studies, extending over several years, by Simpson and co-workers (Simpson, Long \& Byun 2002; Byun, Simpson \& Long 2004; Ma \& Simpson 2005; Byun \& Simpson 2006), using elaborate LaserDoppler Velocimetry (LDV) and Hot-Wire Anemometry (HWA) techniques, the former implemented in a manner allowing the near-wall flow to be mapped with especially high accuracy. Due to the virtual uniqueness of the data set emerging from this work, the flow has increasingly been regarded, over the past 3-4 years, as a key three-dimensional test case for prediction procedures, and there have been several RANS and LES studies that have investigated the ability of the respective methods to capture mainly the mean-flow properties. The Reynolds number of the flow, based on hill height and free-stream velocity, is 130000 , hence bordering on the range of practically relevant flows. The flow exhibits all the complex features peculiar to separation from curved three-dimensional surfaces, including strong skewing of the approaching boundary layer, separation on the leeward of the hill with formation of focal points, reattachment and development of a three-dimensional wake with streamwise vortices.

Several attempts to compute this flow with various RANS methods and models, whether undertaken in a steady or an unsteady mode, have all yielded a poor representation of even the basic mean-flow features, all displaying qualitatively similar defects. For example, Wang, Jang \& Leschziner (2004) report an extensive study with various elaborate nonlinear eddy-viscosity and second-moment-closure models, all giving seriously excessive separation, insufficient rate of post-reattachment recovery and wrong flow structure downstream of the hill. Attempts to induce sheddinglike behaviour, within the RANS framework, through the introduction of periodic excitation in the inlet flow invariably led to a steady flow after the excitation ceased. Similarly, unsuccessful RANS results were also reported by Persson et al. (2006); Benhamadouche et al. (2005) and Visbal, Rizzetta \& Mathew (2007). Viewed against experience gained from studies of two-dimensional separation, this serious failure of even the most elaborate RANS models in this case is surprising, at least at first sight, because the closed recirculation zone is thin, hugging the leeward side, and thus expected not to be dominated by large-scale dynamics. However, the simulations reported herein reveal very complex physics associated with strong three-dimensional 
straining, and suggest that the separation behaviour is very sensitive - considerably more so than in nominally two-dimensional separation - to the resolution of, and details in, the boundary layer as it undergoes separation.

Over the past 3-4 years, about a dozen studies preceding the present one have applied large-eddy simulation and hybrid LES-RANS schemes to this flow. Two in the latter category are those of Davidson \& Dahlströhm (2005) and Tessicini, Li \& Leschziner (2007), both intentionally adopting coarse grids in an effort to demonstrate the economic advantages derived from the respective hybridization techniques. The study by Tessicini et al. (2007), employing a two-layer zonal strategy, contrasts simulations on grids as coarse as 1.5 million nodes against a pure LES performed with 10 million nodes - a density considerably higher than that used later in a pure LES by Patel \& Menon (2007), reviewed below in greater detail, but one that is acknowledged by Tessicini et al. to be significantly lower than that needed to achieve adequate near-wall resolution. Also, in contrast to almost all other studies, including that of Davidson \& Dahlströhm, Tessicini et al. generated a faithful spectral representation of the inlet condition by performing precursor boundary-layer simulations and feeding the data into the flow inlet plane well upstream of the hill. Whilst the zonal scheme and Davidson \& Dahlströhm (2005) hybrid approach are demonstrated to yield a fairly good mean-flow description of the flow on non-wall-resolving coarse grids, judged mainly by reference to the experimental data, the results are pertinent principally to the aim of simulating complex separation in practical configurations at 'acceptable' accuracy and economy, in which case the fundamental physical mechanisms are not at the centre of attention.

In a series of inter-related publications, Patel, Stone \& Menon (2003); Patel \& Menon (2007); Fureby et al. (2004) and Persson et al. (2006) report simulations for the same hill - in the case of Persson et al. (2006), not only with pure LES, but also with Detached Eddy Simulation (DES) and RANS closures, the last already mentioned above. A representative statement on all these simulations may justifiably be made by reference to the most recent publication from this group of collaborators by Patel $\&$ Menon (2007). This reports a pure LES simulation on a grid of 5 million nodes, which is regarded by the writers as rather coarse, as noted already by reference to the simulations of Tessicini et al. (2007). Although nominally wall-resolving, the wall-nearest computational layer of nodes is at $y^{+}>4$ throughout, and the spanwise resolution is especially coarse, the cell-aspect ratio reaching 100 at the hill wall. Moreover, the inlet conditions do not include a realistic prescription of the unsteady features of the boundary layer and the computation domain is rather short. Globally, the results agree reasonably well with the experimental data, but closer inspection reveal not insignificant defects in pressure distribution, the conditions upstream of the mean separation line (which are essentially non-turbulent), the position of separation at the spanwise mid-plane and the predicted topology, which is compared to oilflow measurements recently declared by Simpson as being unreliable and inconsistent with near-wall-velocity LDV measurements. The writers thus regard these simulations as distinctly under-resolved and as providing, essentially, a qualitative view of this complex flow.

Another very recent study is that of Visbal et al. (2007). This included both $k-\varepsilon$ RANS computations and Implicit LES (ILES) simulations - that is, without the use any subgrid-scale model. Instead, dissipation is provided by the numerical method. An important distinction relative to all other studies is that the Reynolds number of the flow simulated was half of the experimental value, a choice made in order "to attain better numerical resolution". The finest mesh used in the simulation contained 
31 million nodes, and this may justifiably be regarded as reasonably wall-resolving and having appropriate cell-aspect ratio. Because of the lower Reynolds number of the flow simulated, the comparisons with the experimental data must be viewed with caution. Although the flow is represented reasonably well, in terms of general trends, there is a rather poor agreement in respect of the pressure in the separated region, the flow topology on the hill surface is not well reproduced, and insufficient information is provided - for example, in respect of turbulence properties - to allow the simulation to be reliably assessed. One interesting result to highlight, however, is that the experimentally observed peak in turbulence energy upstream of the recorded separation line, very close to the wall, is reproduced qualitatively by the simulation. This observation provides a clear indication that near-wall resolution is very important for capturing the fundamental characteristics of the near-wall flow.

The most recent study on the effort to simulate the flow under consideration is by Krajnovic (2008). This study involves the application of a conventional LES second-order finite-volume scheme with the fixed-constant Smagorinsky model over a mesh of around 15 million nodes. Although this grid allowed the wall layer to be resolved down to $y^{+}=0.5$, the cell-aspect ratio near the wall reached values as high as 250 , rendering the near-wall grid extremely anisotropic. Turbulent inlet conditions were approximated by rescaling the solution of an unrelated channel-flow simulation at $R e_{\tau}=500$. Agreement achieved with the experimental observations was rather mixed. Significant differences arose, for example, in respect of the topology, the wake structure, especially the transverse velocity component, the thickness of the recirculation region and, associated with the latter, the pressure field. In particular, as a consequence of the excessively thick and (to a lesser extent) long recirculation zone predicted by the simulation, the pressure variation in the separation zone was seriously mis-represented. Thus, in several respects this solution is inferior to earlier solutions, some produced with considerably coarser grids.

The present paper provides a combined exposition of two independent LES studies on fine, wall-resolving grids for the experimental Reynolds number, not only investigating the level of agreement with corresponding experimental data, but also elucidating links between structural and unsteady features, and statistical information extracted from both simulations. The exposition includes some budgets components in critical locations, spectra and results highlighting unsteady features and structures. The two studies have been pursued independently for about two years, with some principal results recently presented in independent papers at the 11th European Turbulence Conference by Li \& Leschziner (2007) and García-Villalba et al. (2007). It is following this initial publication that the decision was taken to proceed with the present combined manuscript. The two simulations employed different numerical procedures, different grids and slightly different approaches for specifying boundary conditions. The respective grids contained 36.7 million and 134.5 million nodes. While the latter unquestionably provides a better resolution than the former, the relative level of resolution is not quite as large as is implied purely by the ratio of $134.5 / 36.7$, as shown in $\S 3.2$. Both simulations are wall-resolving, adhering carefully to accepted cell-aspect ratios and expansion rates and involving very careful specifications of the unsteady inlet conditions. There are several merits to this combined presentation: first, it allows the level of confidence in the computed results to be judged more reliably; second, it permits a more comprehensive picture of the physics to be conveyed; and third, it illustrates in some important respects the sensitivity to grid resolution within an unprecedented range of grid density. Apart from providing insight into important fundamental physical phenomena associated with separation from curved surfaces, 
the study arguably offers, alongside the experimental data, a solid benchmark and yardstick for assessing the capabilities of other computational techniques.

\section{Experimental configuration and summary of findings}

The experimental configuration under consideration consisted of a threedimensional axisymmetric hill mounted in the spanwise centre of a $0.25 \mathrm{~m}$ high duct having a length of $7.62 \mathrm{~m}$ and width of $0.91 \mathrm{~m}$. The hill shape is described by

$$
\frac{y(r)}{H}=-\frac{1}{6.04844}\left[J_{0}(\Lambda) I_{0}\left(\Lambda \frac{r}{2 H}\right)-I_{0}(\Lambda) J_{0}\left(\Lambda \frac{r}{2 H}\right)\right],
$$

where $H=78 \mathrm{~mm}$ is the hill height, $\Lambda=3.1926, J_{0}$ is the Bessel function of the first kind while $I_{0}$ is the modified Bessel function of the first kind. The nominal free-stream velocity in the duct was $U_{\text {ref }}=27.5 \mathrm{~m} \mathrm{~s}^{-1}$, and the free-stream turbulence intensity was $0.1 \%$. The Reynolds number based on $U_{\text {ref }}$ and $H$ is 130000 . In the absence of the hill, a zero-pressure-gradient turbulent boundary layer of thickness $\delta=0.5 H=39 \mathrm{~mm}$ was present on the upper and lower walls of the tunnel at the hill-crest position, its momentum-thickness Reynolds number being $R e_{\theta}=7300$.

Various parts or stages of the experimental campaign are reported in Simpson et al. (2002); Ma \& Simpson (2005); Byun \& Simpson (2006), utilizing a variety of experimental techniques, including LDV, HWA, pressure sensors and oil-flow visualization to study different aspects of the flow. In the first paper, Simpson et al. (2002) report mean surface pressures, obtained by means of multiple pressure taps, mounted flush with the surface, oil flow visualizations and three-component LDV measurements in the near wake of the hill. These measurements conveyed an initial description of the principal flow features. Accordingly, the flow decelerates in front of the hill, without separating (in the mean), and then accelerates over and around the hill, with the fluid at increasing spanwise distance from the centre-plane deflected sideways. This gives rise to two streamwise counter-rotating vortices in the wake, which may be viewed as the two legs of a horseshoe vortex. The flow separates from the leeward of the hill, the oil-flow visualization suggesting a very complex, multiplevortex pattern with two main separation regions, a small one between $x / H \sim 0.18$ and $x / H \sim 0.4$ and a large one covering the region $x / H \sim 0.4$ to $x / H \sim 2$, accompanied by a high adverse pressure gradient. However, subsequent experimental studies of the near-wall flow, undertaken with high-resolution LDV, negated the initial interpretation of three pairs of vortices derived from the oil-flow experiments. This contradiction will be considered below. Following reattachment at $x / H \sim 2$, the flow undergoes a recovery, and the wake is characterized by a perturbed boundary layer containing the pair of large streamwise vortices mentioned earlier.

The downstream wake development was studied later by Ma \& Simpson (2005), using a four-sensor hot-wire probe. The three velocity components were measured behind the hill at three cross-sections $x / H=2.62,3.63$ and 6.59. Spectra and two-point correlations were also reported. One of the measurement planes, $x / H=3.63$, coincided with the LDV measurement plane reported by Simpson et al. (2002). Significant discrepancies were observed between the two sets of measurements. For example, in the symmetry plane, a much higher level of turbulence energy was obtained from the LDV measurements than from the hot-wire measurements in a region far removed from the wall (at $y / H>0.5$ ). In a personal communication, Simpson (2006, personal communication) acknowledged problems with seeding in the outer region, possibly invalidating the LDV measurements in that region. Consequently, at $x / H=3.63$, the 
data derived from the hot-wire measurements must be regarded as far more secure. It should be mentioned here that Persson et al. (2006); Visbal et al. (2007); Patel \& Menon (2007) and Krajnovic (2008) all compared their results in the wake to the less reliable LDV data.

The hot-wire data confirmed the presence of two large, wall-normal flattened, counter-rotating streamwise vortices, which originate from the reorientation of the vorticity of the upstream boundary layer. Closer inspection of the secondary motions close to the symmetry plane indicated the presence of two additional small counterrotating vortices close to the wall, at $y / H<0.4$ (see figures 19-20 below). A peculiar feature revealed by long-duration experiments was the presence of low frequency meandering motions, which make a non-negligible contribution to the time-averaged turbulent kinetic energy.

Finally, Byun \& Simpson (2006) reported highly space-resolved LDV measurements very close to the surface on the leeside of the hill. In the near-wall region (within $1 \mathrm{~cm}$ ), a miniature LDV probe was used, while in the outer region a conventional LDV system was employed. The analysis of the mean flow in the symmetry plane showed that the flow accelerates past the crest region and then decelerates near the wall as it is subjected to an adverse pressure gradient, reaching a stagnation point at about $x / H \sim 0.96$. Beyond that location, there exists a long and thin backflow region (see figure 12 below). The LDV data very close to the wall allowed the hillsurface topology to be constructed, and this yielded a very different pattern to that derived from the oil-flow visualization. The differences were attributed to the effect of gravity on the oil flow, to the influence of unsteady forces on the finite-thickness oil mixture and to the shear stress of the back flow. The small separation region between $x / H \sim 0.18$ and $x / H \sim 0.4$ observed in the oil-flow study was not obtained in the LDV measurements. Moreover, the spatial structure of the main separation region was deduced to be simpler than that suggested by the oil-flow visualization, displaying only one pair of counter-rotating vortices (see figures 15-17 below). An intriguing feature observed close to the wall at $x / H \sim 0.3$, roughly midway between the hill crest and the separation line, was the presence of a high-level turbulence-energy region. This region corresponds, approximately, to the location of a very high adverse pressure gradient reported by Simpson et al. (2002), suggesting fully attached flow in the mean but with a very high level of fluctuations. Yet another peculiar feature observed was the existence of bimodal velocity PDFs in the separation region (Byun 2005). This was interpreted as reflecting a low-frequency meandering of the flow field, and it was further speculated that the bimodal features may be indicative of intermittent separation patterns involving a switching between detached and attached flow.

\section{Computational details}

\subsection{Equations, numerical solution procedure and subgrid-scale model}

Both computational schemes solve the following implicitly filtered Navier-Stokes equations governing incompressible, constant-property flow for the resolved velocity $\overline{u_{i}}$ and the resolved (kinematic) pressure $\bar{p}$ :

$$
\begin{gathered}
\frac{\partial \overline{u_{i}}}{\partial x_{i}}=0 \\
\frac{\partial \overline{u_{i}}}{\partial t}+\frac{\partial \overline{u_{i}} \overline{u_{j}}}{\partial x_{j}}=-\frac{\partial \bar{p}}{\partial x_{i}}+\frac{\partial\left(2 \nu \overline{S_{i j}}\right)}{\partial x_{j}}-\frac{\partial \tau_{i j}}{\partial x_{j}}
\end{gathered}
$$


where $v$ is the molecular viscosity and $\overline{S_{i j}}=\frac{1}{2}\left(\partial \overline{u_{i}} / \partial x_{j}+\partial \overline{u_{j}} / \partial x_{i}\right)$ is the filtered strain-rate tensor. The subgrid-scale stresses $\tau_{i j}=\overline{u_{i} u_{j}}-\overline{u_{i}} \overline{u_{j}}$ result from the unresolved motions filtered out by the numerical grid, and their modelling is discussed later.

Two entirely independent codes have been used to obtain the results presented below. Both solve (3.1) and (3.2) on body-fitted, curvilinear grids using a cell-centred finite-volume method with collocated storage of the Cartesian velocity components. Second-order central differences are employed for the convection as well as for the diffusive terms.

The simulation of García-Villalba \& Rodi (henceforth GVR; University of Karlsruhe team), was performed with the in-house code LESOCC2. This is a successor of the code LESOCC (Breuer \& Rodi 1996), and its most recent version is described by Hinterberger (2004). A fractional step method is used, comprising a Runge-Kutta predictor and the solution of pressure-correction equation as a corrector (Le \& Moin 1991). The Rhie and Chow momentum-interpolation method (Rhie \& Chow 1983) is applied to avoid pressure-velocity decoupling. The Poisson equation for the pressure correction is solved iteratively by means of the 'strongly implicit procedure' (Stone 1968). Parallelization is implemented via domain decomposition, and explicit message passing is used with two halo cells along any inter-domain boundary for intermediate storage.

The procedure of $\mathrm{Li} \&$ Leschziner (henceforth LL; Imperial College team), is implemented in the in-house code STREAM-LES, initially developed by Lardat \& Leschziner (1998). Time-marching is based on a fractional-step method. The flux terms are advanced explicitly using the second-order Gear or the third-order AdamsBashforth method. The provisional velocity field is then corrected by projecting the pressure gradient onto a divergence-free velocity field by solving the pressurePoisson equation with a three-dimensional V-cycle multigrid algorithm, operating in conjunction with a successive line over-relaxation scheme. This code is fully parallelized using MPI by way of domain decomposition, although the halo-cell arrangement is slightly different from that in the LESOCC2 code.

Both groups have used the dynamic Smagorinsky subgrid-scale model, first proposed by Germano et al. (1991) and subsequently modified by Lilly (1992). This models the anisotropic part of the SGS term via

$$
\tau_{i j}-\frac{1}{3} \delta_{i j} \tau_{k k}=-2 v_{s g s} \overline{S_{i j}}
$$

while the trace $\tau_{k k}$ is lumped into a modified pressure. The subgrid-scale viscosity is given by

$$
v_{s g s}=C \Delta^{2}|\bar{S}|, \quad|\bar{S}|=\left(2 \overline{S_{i j}} \overline{S_{i j}}\right)^{1 / 2}
$$

with $\Delta=(\Delta x \Delta y \Delta z)^{1 / 3}$. In the dynamic model, the parameter $C$ is determined using an explicit box filter of width equal to twice the mesh size. The two groups used different approaches for smoothing the variation of $C$. In the GVR procedure, the subgrid-scale viscosity $v_{\text {sgs }}$ was first clipped to avoid negative values and then $C$ was smoothed by the temporal under-relaxation (Breuer \& Rodi 1996)

$$
C^{n+1}=\varepsilon C^{*}+(1-\varepsilon) C^{n},
$$

with the relaxation factor $\varepsilon=5 \times 10^{-4}$ and $C^{*}$ the value determined by the original model.

Following preliminary test simulations on a 3.5 million grid, undertaken with a zonal LES-RANS scheme and employing the alternative practices of temporal smoothing 


\begin{tabular}{lcccc} 
Group & \multicolumn{1}{c}{ Code } & $L x \times L y \times L z$ & $N x \times N y \times N z$ & $T_{a v}$ \\
LL & STREAM-LES & $13 H \times 3.2 H \times 11.7 H$ & $512 \times 160 \times 448$ & $150 \mathrm{H} / \mathrm{U}_{\text {ref }}$ \\
GVR & LESOCC2 & $20 H \times 3.2 H \times 11.7 H$ & $770 \times 240 \times 728$ & $240 \mathrm{H} / U_{\text {ref }}$
\end{tabular}

TABle 1. Parameters of the simulations.

and spanwise averaging, the latter method was adopted by LL despite the lack of spanwise homogeneity, principally in view of the observed very minor sensitivity to the smoothing practice even on this much coarser grid than the one actually used. Both approaches employed could be criticized: spanwise averaging because of the lack of homogeneity, and temporal relaxation if note is taken of the fact that the all-important separation process varies substantially in time over time scales that exceed $H / U_{\text {ref }}$. Temporal relaxation, however, reflects the notion of homogeneity in time. With the fine grids used, especially by GVR, the contribution of subgrid-scale stresses may be assumed to be negligible. While this is, indeed, the case over most parts of the flow, it is shown in $\S 3.5$ that the subgrid-scale viscosity is not negligible in the highly turbulent separated mixing layer.

In what follows, the overbars that denote the resolved quantities will be omitted for simplicity.

\subsection{Computational domain, grid and boundary conditions}

A sketch of the computational domain used by GVR is shown in figure 1, the dimensions of which are given in table 1 . The cross-sectional area of the experimental duct, $3.2 \mathrm{H} \times 11.7 \mathrm{H}$, is resolved by both simulations. Also, in both simulations the inflow plane is placed at $x / H=-4$. The domain of GVR extends downstream to $x / H=16$, in line with earlier simulations, while that of LL is restricted to $x / H=9$, so as to reduce the computational cost. Although the domain is longer in GVR's simulation, a relatively small number of grid points (in streamwise direction) are located between $x / H=9$ and $x / H=16$ because the region of interest is the near wake up to, say, $x / H=5$. In view of the fact that, in the experiment, post-reattachment recovery starts at $x / H \sim 2$ and that the maximum mean transverse velocity is only $10 \%$ of the free-stream value at $x / H=3.63$, both domains are regarded as entirely adequate.

Table 1 also provides the number of grid cells used in both simulations. The GVR mesh contains 134.5 million nodes (excluding the additional grid used for generating the inflow), while the LL mesh contains 36.7 million nodes, but over a shorter domain. In both cases the choice of the grid is based on experience gained with preliminary calculations on coarser grids. Two views of the symmetry-plane grid used by the GVR group are shown in figure $2(a-b)$. As the GVR mesh covers a larger domain, the resolution of the two meshes is not as different as indicated by the total number of points. The GVR grid is finer by a factor of approximately 2 in the streamwise and spanwise directions close to the hill $(|x / H|<4,|z / H|<2)$. In the wall-normal direction, however, the LL grid is somewhat finer below $y / H=0.02$, but stretches at a larger ratio towards the outer flow. This is shown in figure $2(d)$, which displays the grid spacing in the wall-normal direction very close to the bottom wall. The stretching rate of the grid in that direction is $1.7 \%$ in the GVR grid and $3 \%$ in the LL grid. Another difference is that the GVR grid is nearly orthogonal in the near-wall region, while the height-wise grid lines of the LL grid are straight and normal to the upper 

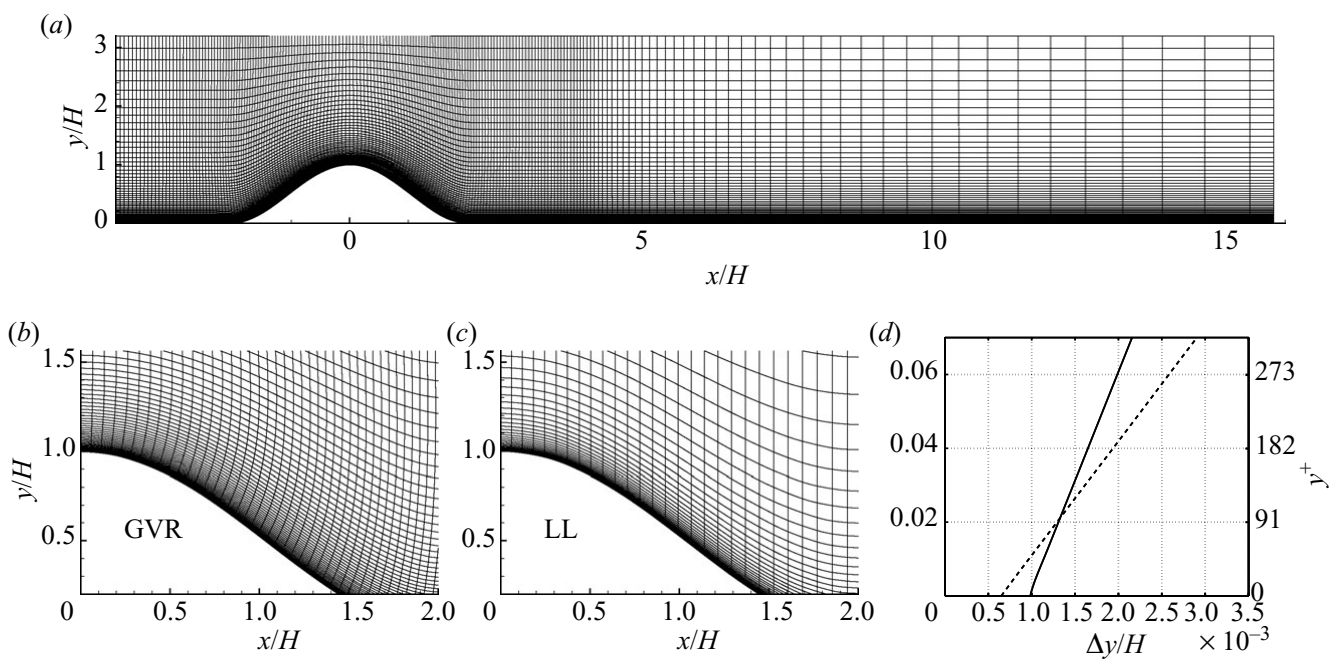

FiguRE 2. Grid in the symmetry plane. $(a-c)$ Two views of the grids. Every fourth grid line is shown. ( $a-b)$ GVR grid. (c) LL grid. (d) Comparison of grid-spacing in wall-normal direction between both grids (zoom close to the wall). Solid line, GVR grid; dashed line, LL grid.

wall. However, orthogonality is not an issue in either of the procedures used, as both are based on general non-orthogonal finite-volume implementations with Cartesian velocity decomposition.

The quality of the grid resolution may be judged by focusing on the grid spacings in wall units. Figure 3 displays, for both grids, contour plots of the distance between the centroids of the wall-adjacent cells and the wall, $y_{1}^{+}$, and the streamwise and the spanwise cell sizes in wall units, defined with the local friction velocity. In GVR's grid, the value of $y_{1}^{+}$is around 2 over most of the domain, except for the windward part of the hill where the flow accelerates strongly, hence leading to locally larger friction velocity and thus larger values of $y_{1}^{+}$. As is evident from figure 3 , the near-wall resolution in LL's grid is slightly better, as the wall distance $y_{1}^{+}$is below 1.8 , even in the high-shear region on the windward of the hill. The streamwise and the spanwise cell dimensions in GVR's simulation, again in wall units, are roughly 70 and 30, respectively - except in the front part of the hill. The LL grid is approximately twice as coarse as GVR's grid in both directions.

Both groups employed a no-slip condition at the bottom wall, as the wall-nearest cell lies within the viscous sub-layer, as pointed out earlier. The frictional boundary condition at the upper wall (as contrasted with the impermeability condition) is of subordinate importance, because this wall is located far from the region of interest. Hence, neither group attempted a full resolution of the boundary layer on that wall, opting for an analytical wall-law representation instead, to bridge the viscous nearwall region. The GVR team used the 1/7th power-law-based wall function proposed by Werner \& Wengle (1993), while LL implemented a standard log-law formulation, both of which are very similar in principle.

At the lateral walls, GVR imposed free-slip conditions, while LL used the same wall function as that applied to the upper wall. Again, these boundaries are far from the region of interest, and the difference are expected to have a negligible effect on the solution. 

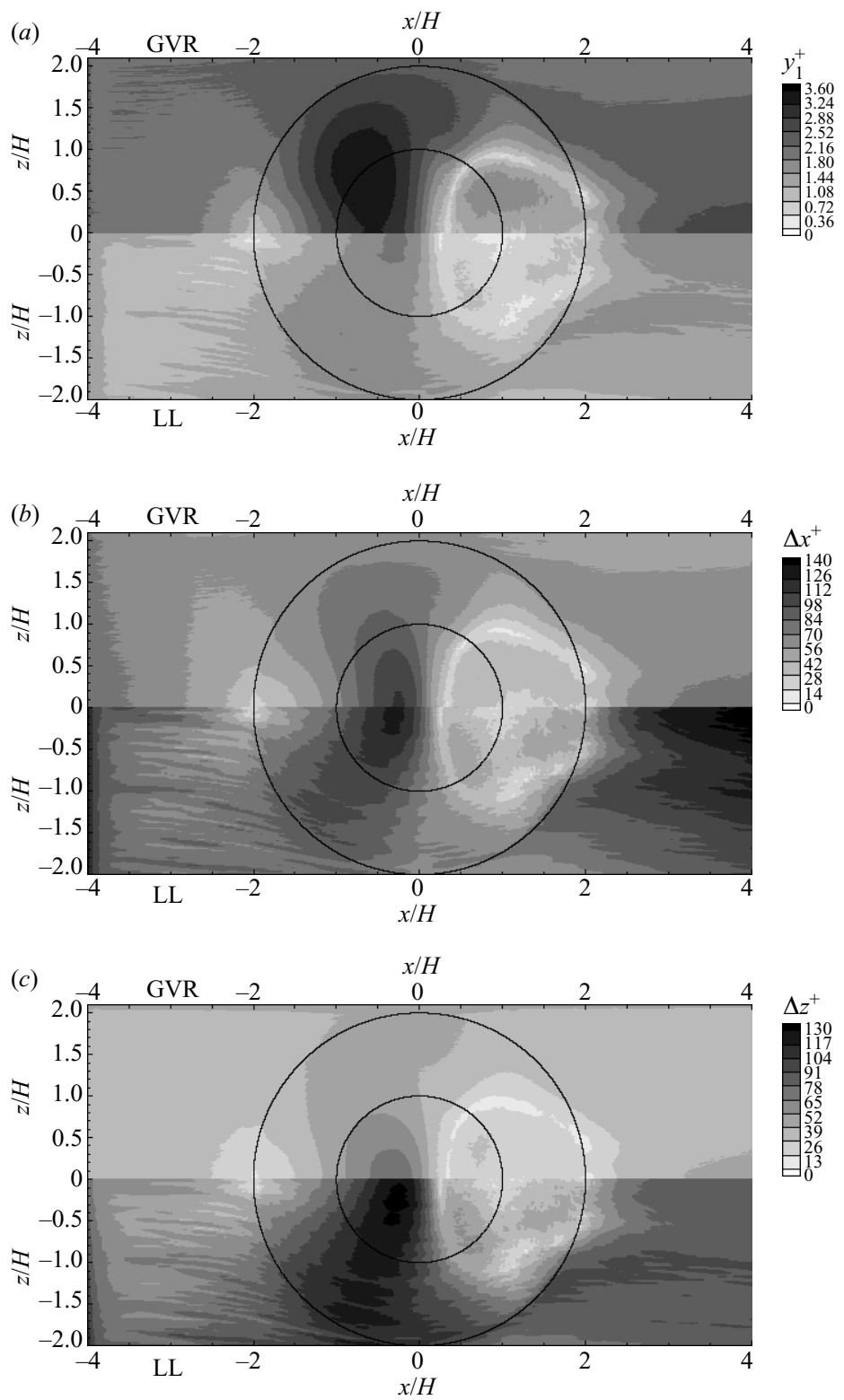

FigURE 3. Grid spacings in wall units. Upper half of each subfigure, GVR. Lower half, LL. (a) $y_{1}^{+} ;\left(\right.$b) $\Delta x^{+} ;$(c) $\Delta z^{+}$.

At the exit plane, both teams used convective outflow conditions. The inflow conditions are of major importance, require an elaborate procedure to secure compliance with the experiments and are discussed separately below.

\subsection{Generation of inflow conditions}

As noted in $\S 2$, the experimental flow approaching the hill contains a relatively thick turbulent boundary layer, extending to approximately $50 \%$ of the hill height. Hence, an accurate description of the inflow, both in terms of its statistical properties and the unsteady turbulent structures, is essential if confidence is to be placed in the solution. 
The methods used by both groups to generate the inlet conditions are slightly different. In both cases, precursor simulations were performed, in combination with a method developed by Pierce (2001) that allows the turbulence statistics of a simulation of spatially periodic channel to be matched to arbitrarily prescribed statistics, using a corrective body-force technique. Data from these precursor simulations were then fed in at the inflow plane.

In GVR's implementation, the precursor simulation progressed simultaneously with the main simulation, while LL separated the precursor from the main simulation, performing first a quasi-periodic boundary-layer simulation, subject to experimental constraints, and then storing a series of realizations covering 7.2 time units $H / U_{\text {ref }}$. The start and end of this series were then modified so as to ensure continuity in the loop formed by connecting the end to the beginning, allowing the loop to be repeated for as long as the main simulation required. While this practice inevitably introduces a periodic component associated with the length of the loop, the period is very different from the time scale of the flow.

The velocity and turbulent-stress profiles to be matched are those measured by Simpson et al. (2002) in the wind tunnel when the hill was not in place. The approach of GVR was to force the precursor simulation to match the prescribed mean streamwise velocity profile only, achieved by adding to the streamwise-momentum equation the following forcing term at every time step:

$$
f_{x}(y, z, t)=-\langle u\rangle_{x}(y, z, t)+T(y, z),
$$

where $f_{x}$ is the body force in the $u$-momentum equation, $T$ is the target mean streamwise-velocity profile and $\langle\cdot\rangle_{x}$ indicates averaging over the streamwise direction only. The length of the duct portion used for the precursor simulation was $L_{i n f}=$ $1.8 H=3.6 \delta$, and the number of cells in streamwise direction was 110 . The cost of the precursor simulation was thus around $1 / 8$ th of the total cost. It must be acknowledged that this practice also introduces a periodic component into the main simulation because of the limited length of the periodic duct portion used for the precursor simulation. In this case, periodicity at the frequency $f_{\text {inf }}=U_{\text {ref }} / L_{\text {inf }}=0.556 U_{\text {ref }} / H$ was observed to be present in the spectra of the velocity fluctuations in the main domain. However, only in regions having a negligible turbulence level, e.g. in the free stream, a distinct peak in the spectra was observed, as discussed in $\S 5.2$.

The LL forcing practice also included matching to the streamwise normal stress, in addition to the mean velocity. This forcing was effected by

$$
u(x, y, z, t) \rightarrow \frac{T^{\prime}(y, z)}{\sqrt{\overline{{u_{x}^{\prime 2}}_{x}}(y, z, t)}}\left[u(x, y, z, t)-\langle u\rangle_{x}(y, z, t)\right]+T(y, z),
$$

where the velocity fluctuations were first rescaled according to the ratio of the targeted streamwise root mean square (r.m.s.) velocity, $T^{\prime}(y, z)$, and the computed r.m.s. value, before being added to the mean target velocity, $T(y, z)$. The method involves a first iteration in the precursor simulation that estimates the $T^{\prime}(y, z)$ distribution, based on the available experimental turbulence-energy distribution (the estimate based on the assumption of isotropy), followed by a second iteration in which both the mean velocity and the streamwise normal stress are forced.

In summary, as demonstrated in the comparisons given in figure 4, both methods result in realistic inflow conditions that are judged to be distinctly superior to any practice previously adopted in simulations for this flow. In both cases, the mean flow is matched identically by definition. The turbulence energy is in good agreement in 
(a)

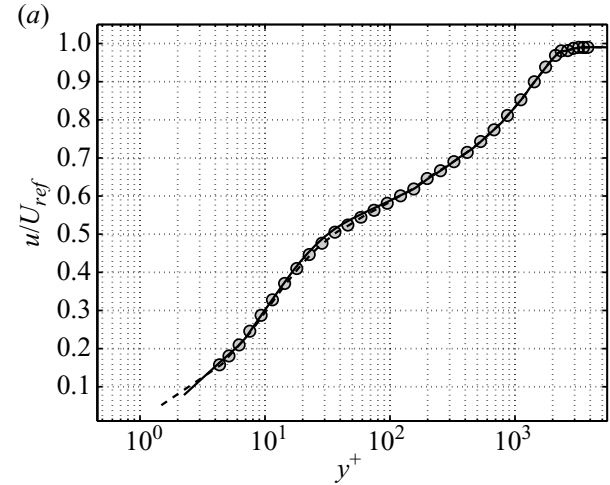

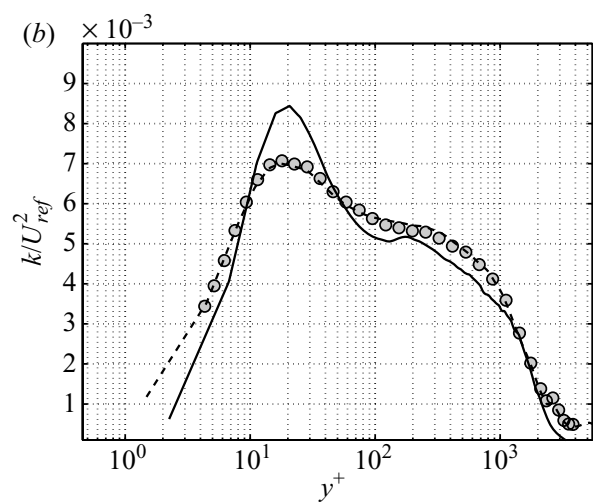

FIgURE 4. Profiles at the inlet obtained from the precursor simulation. (a) mean streamwise velocity $\langle u\rangle / U_{r e f}$. (b) turbulent kinetic energy $k / U_{r e f}^{2}$. Solid line, GVR; dashed line, LL; symbols, experimental data.

both cases - slightly better in the LL simulation because of the additional forcing. Most importantly, in both cases the inflow possesses realistic turbulent length and velocity scales, as well as the appropriate spectrum of scales for the real Reynolds number.

\subsection{Convergence history}

Throughout the paper, reference quantities for length, velocity and time are $H, U_{\text {ref }}$ and $H / U_{\text {ref }}$, respectively. Unless otherwise stated, all data presented are normalized with these quantities. At some locations, profiles are reported in wall units, which are based always on the friction velocity of the boundary layer at the inflow plane. After discarding initial transients, averages were collected over 240 and 150 time units $H / U_{\text {ref }}$ in the GVR and LL simulations (see table 1), respectively. Additionally, the symmetry of the flow with respect to the centre plane $z=0$ was exploited (or imposed) to compute the mean quantities. This averaging period is sufficiently long to achieve converged fields in respect of the large majority of results, as demonstrated in comparisons to follow. Note that the averaging period is somewhat longer in GVR's simulation, and this leads to slightly smoother statistical quantities. Figure 5 displays the evolution in time obtained by GVR of the mean streamwise velocity and the turbulent kinetic energy $k$ in the near wake of the hill at two locations that are symmetric with respect to the hill centre plane. Both $\langle u\rangle$ and $k$, recorded at the two symmetric locations, converge to the same respective levels as expected, and differences are small after 100 time units. The occasional excursions in the evolution of $k$ indicate the occurrence of very energetic events. This issue is revisited in the discussion later (§5.2).

\subsection{Evaluation of subgrid-scale model contribution}

Even with the fine grids used in this investigation, the subgrid-scale viscosity returned by the dynamic model is not negligible in regions of high level of turbulence. This can be seen in figure 6 which displays contours of time-averaged subgrid-scale viscosity $\left\langle v_{s g s}\right\rangle$ normalized by the molecular viscosity $v$ in the symmetry plane. The maximum values of $\left\langle v_{s g s}\right\rangle / v$ are roughly 6 in GVR and 18 in LL, and they are attained in the separated shear layer at $x / H \sim 0.4-1$. Away from this location, the values are much lower. The differences in the viscosity levels mainly reflect corresponding differences in 

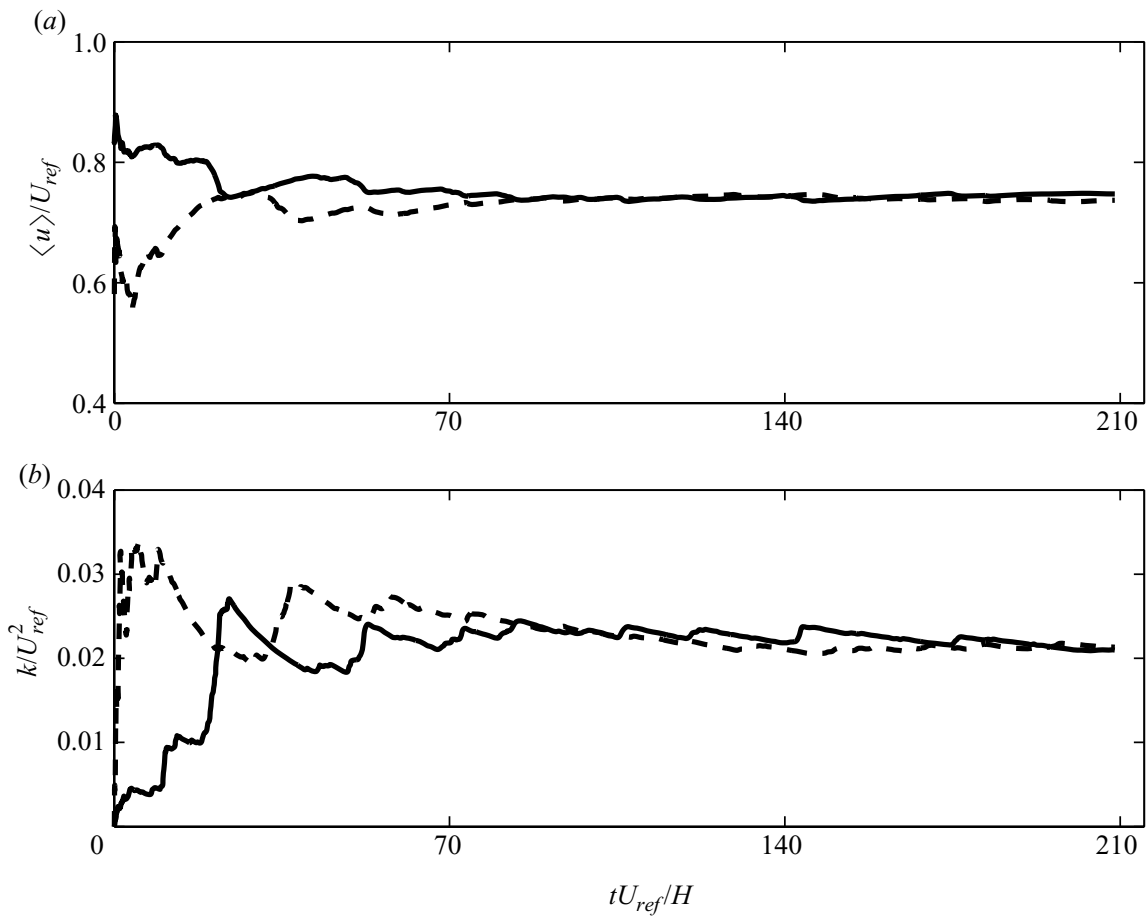

Figure 5. Evolution in time of the mean streamwise velocity $(a)$ and the turbulent kinetic energy $(b)$ at $x / H=2.62, y / H=0.23$ from GVR. Solid line, $z / H=0.84$; dashed line, $z / H=-0.84$.
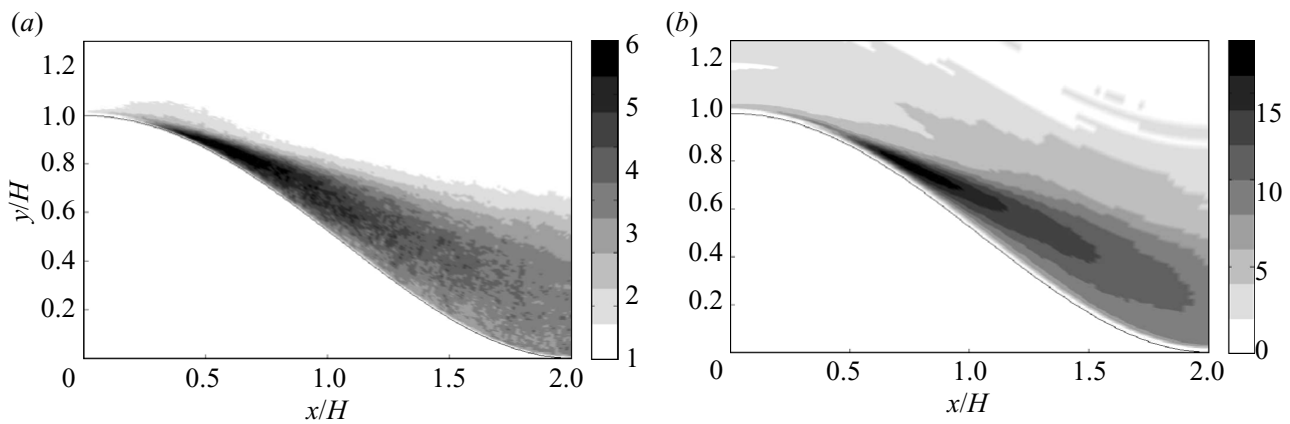

FIGURE 6. Time-averaged subgrid-scale viscosity $\left\langle v_{s g s}\right\rangle / v$ in the symmetry plane. $(a)$ GVR; (b) LL.

the filter size $\Delta$ resulting from the different grid resolutions, especially in the spanwise direction.

One of several routes for quantifying the relative importance of the subgrid-scale viscosity is to compare it to an equivalent eddy-viscosity $v_{T}$ defined in a Reynoldsaveraged sense, which represents the effect of the resolved turbulent motion. The linear eddy-viscosity hypothesis states that the deviatoric Reynolds stress is proportional to the mean rate of strain, that is,

$$
-\left\langle u_{i}^{\prime} u_{j}^{\prime}\right\rangle+\frac{2}{3} k \delta_{i j}=2 v_{T}\left\langle S_{i j}\right\rangle
$$



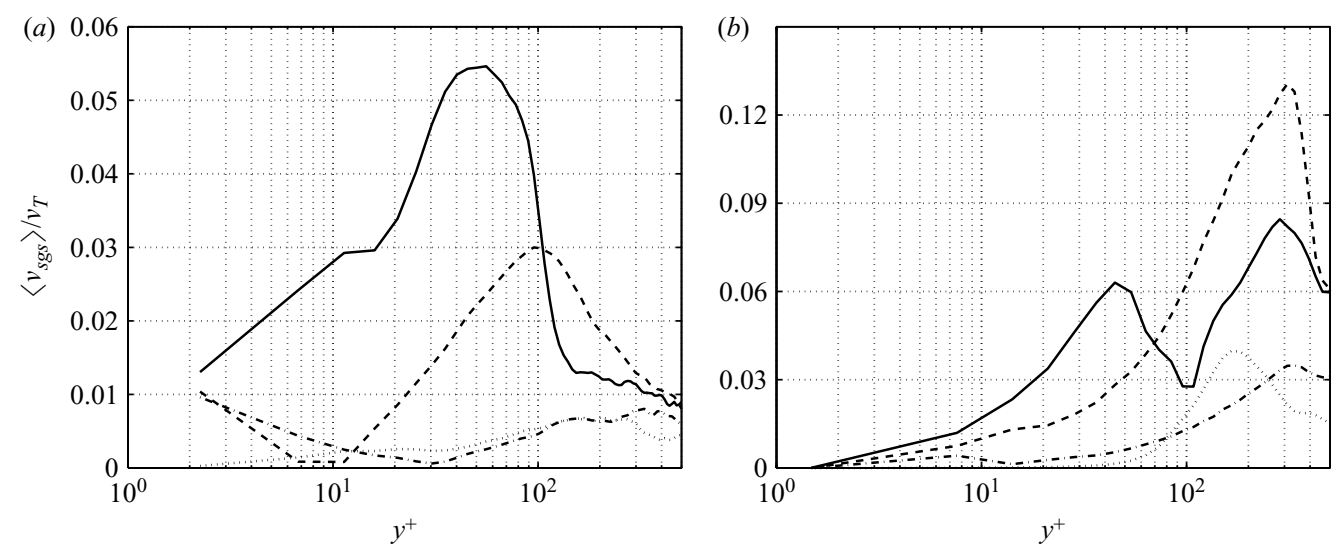

FIGURE 7. Profiles of the ratio between the time-averaged subgrid-scale viscosity $\left\langle v_{s g s}\right\rangle$ and an equivalent total eddy viscosity $v_{T}$ as a function of $y^{+} . z / H=0$. Solid line, $x / H=0.3$. Dashed line, $x / H=0.6$. Dashed-dotted line, $x / H=1$. Dotted line, $x / H=2$. $(a)$ GVR; $(b)$ LL.

It is clearly not possible to extract a unique eddy viscosity directly from the above tensorial relationship. However, following Wissink, Michelassi \& Rodi (2004), a representative eddy viscosity may be evaluated using

$$
v_{T}=\frac{\left\|\frac{2}{3} k \delta_{i j}-\left\langle u_{i}^{\prime} u_{j}^{\prime}\right\rangle\right\|}{\left\|2\left\langle S_{i j}\right\rangle\right\|},
$$

where the norm $\|\cdot\|$ of the matrix $a_{i j}$ is defined by $\left\|a_{i j}\right\|=\sqrt{a_{i j} a_{i j}}$. Figure 7 shows profiles of the ratio $\left\langle v_{s g s}\right\rangle / v_{T}$ as a function of the wall distance, in the leeward of the hill. The profiles are extracted in the symmetry plane at $x / H=0.3,0.6,1$ and 2 . These profiles show that the value of the subgrid-scale viscosity is much lower than the value of the eddy viscosity, as defined by (3.9). Also, the maximum values are seen to occur well away from the wall in the highly turbulent separated shear layer, and are about $6 \%$ and $12 \%$ in GVR's and LL's simulations, respectively, the difference being again due to the finer grid in GVR's simulation, especially in the spanwise direction. These relatively small values indicate, albeit notionally, that the impact of the subgrid-scale model on the solution is likely to be minor.

\section{Results for statistically averaged quantities}

\subsection{General view}

A qualitative description of the flow, based on experimental observations, was provided in $\S 2$. This view is complemented here by a few visualizations derived from the simulations and shown in figures 8 and 9 . The former shows two views of a time-mean flow topology map on the hill surface, while the latter contains two views of the flow visualized by stream traces having respective origins close to the lower wall at different spanwise locations upstream of the hill. The topology map was constructed from the wall-parallel velocity components predicted by the GVR simulation across the wall-nearest computational plane. Figure 3 shows this plane to be at a distance $y^{+}$in the range $0-3.6$, with the value over most of the interesting surface area being 1.0 or less.

Figure $8(b)$ shows the wall topology map for one half of the domain. If both sides of the hill are taken into account, the existence of eight topological features is 
(a)

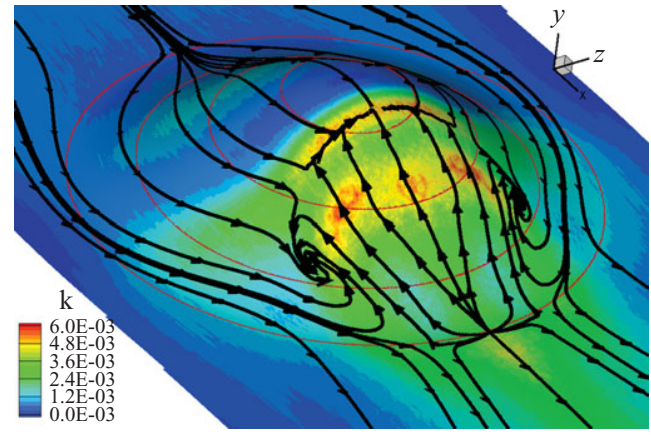

(b)

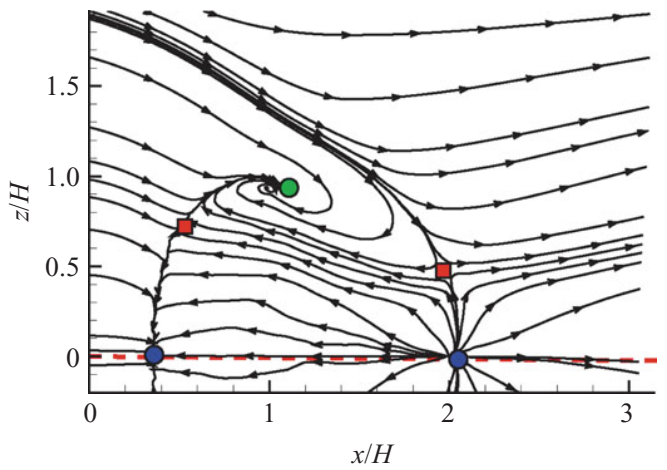

FIGURE 8. Streamlines of the mean flow projected onto the first grid surface from GVR. (a) Three-dimensional view, colour represents turbulent kinetic energy. (b) Two-dimensional view, the dashed red line indicates the symmetry plane.

(a)

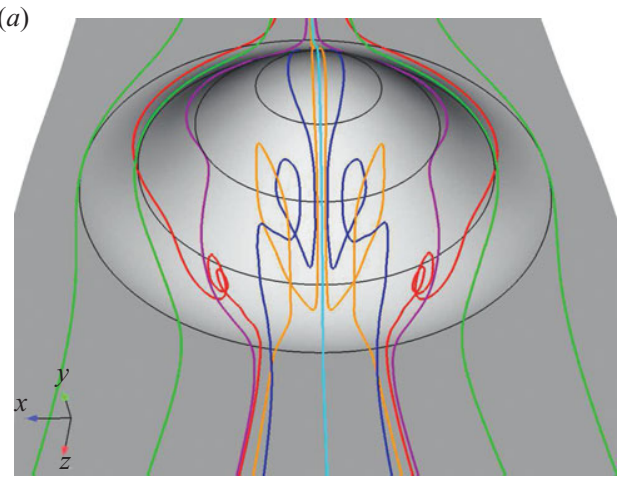

(b)

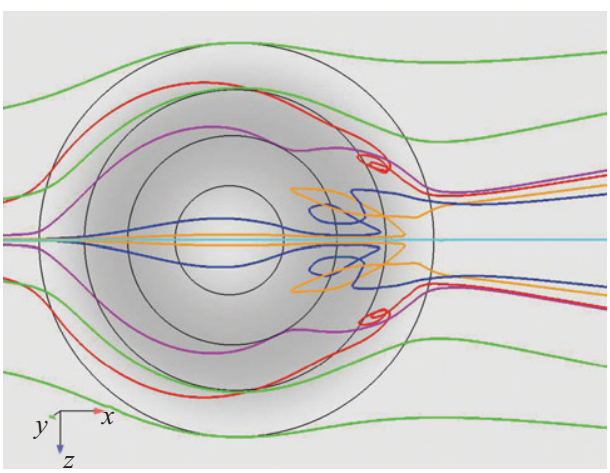

FIGURE 9. Selected streamlines of the mean flow from GVR.

revealed - four saddle points, two identified by red squares, two nodes which are foci, identified by green circles and two nodes which are the separation and reattachment points on the centreline identified by blue circles. The topological features observed satisfy the rules provided by Hunt et al. (1978) for flow over obstacles, namely that the number of saddles points (4) be equal to the number of nodal points $(2+2)$. The saddle points are to be found on either side of the separation and reattachment nodes. The existence of only one pair of vortical foci is emphasized, because this has been the subject of much debate following the publication of oil-flow images by Simpson et al. (2002), which suggested the existence of three pairs of vortical foci, rather than one as reported later in Byun \& Simpson (2006). As shown later, a pair of secondary vortical structures are observed in the flow above the surface, but these are not reflected by corresponding focal points in the wall topology map. Along the inner portion of the separation line - a stretch limited by the two saddle points on either side of the symmetry line - fluid converges towards the node on the centre plane and is then ejected upwards away from the wall. Along the remaining outer portions of the separation line, fluid is converging towards the vortical focus and then shed away from the surface. The vorticity associated with this shedding process can be identified in the wake, which is discussed later. 
The stream traces in figure 9 have been coloured by reference to their upstream origin, so as to identify the association of specific upstream flow portions with characteristic features observed downstream of the hill crest. The outermost two pairs of green traces indicate that the outer flow close to the wall is merely deflected by the hill and is not involved in the separation process. The intermediate pair of red traces, in contrast, point to the flow closer to the hill crest merging into the vortex that is shed from the vortical focus seen in the topology map. The purple lines farther inside avoid this vortical region by first being slightly deflected and then passing over it. Finally, the innermost pairs of blue and orange traces suggest, intriguingly, the presence of two further vortical structures not seen in the wall topology map and having a sense of rotation opposite to that of the vortices shed from the focal points. Upon passing the hill crest, these traces initially move above the very thin upstream nose of the separation line. They are then carried into a 'spiral' present above the wall, ending with an ejection of vorticity into the wake. The origin of this spiral can be traced to the shedding of vorticity from the stable node at the upstream nose of the separation line at which the two arms of the spiral are connected. This process is discussed below by reference to the structure of the flow within and above the separated region. In common with the vorticity shed from the focal point, that originating from the separation node can also be identified in the leeside of the hill as shown in $\S 4.4$.

\subsection{Pressure distribution}

Figures 10 and 11 compare predicted variations of the pressure coefficient across the hill surface, one against the other as well as both with the experimental data (Simpson et al. 2002). Figure 10 provides a full view by means of pressure-coefficient contours. The contours are generally well predicted by both simulations, but there are some differences around the reattachment point. Specifically, the measured value exceeds 0.308 (contour 15), while the simulations, especially GVRs, predict somewhat lower values. The differences become clearer upon inspection of the wall-pressure profiles along the hill centreline given in figure 11, especially the profiles in the magnified leeward portion over which the flow is separated. The LL simulation can be seen to be in good agreement with the experiment, except for the peak pressure at reattachment, while the GVR simulation underestimates the pressure and also the flatness of the plateau. The pressure variation - the shape of the plateau in particular - closely reflects the separation and recovery behaviour as well as the shape of the recirculation region that causes the displacement of the flow above it. As will emerge from a comparison of predicted topology maps in the next section, both simulations predict virtually identical locations for the separation and reattachment nodes on the centre plane. Hence, the implication of the differences in figure 11 is that GVR's simulation predicts a larger rate of thickening of the separated layer in its upstream portion, followed by a smaller rate of thickening in the middle portion of the layer where the inflexion in $C_{p}$ occurs, a lower rate of thinning towards the reattachment node and a slower recovery following reattachment. Upstream of the hill, the pressure coefficient increases as the hill is approached, reaching a local maximum shortly after the windward slope of the hill starts (figure 11a). This maximum is slightly better predicted in the GVR simulation, implying that this simulation captures better the thickening of the flow that arises from the adverse pressure gradient acting on the boundary layer in this region. Thereafter, the flow accelerates and the pressure drops significantly reaching a local minimum at the top of the hill, essentially reflecting the dominance of inviscid processes. 

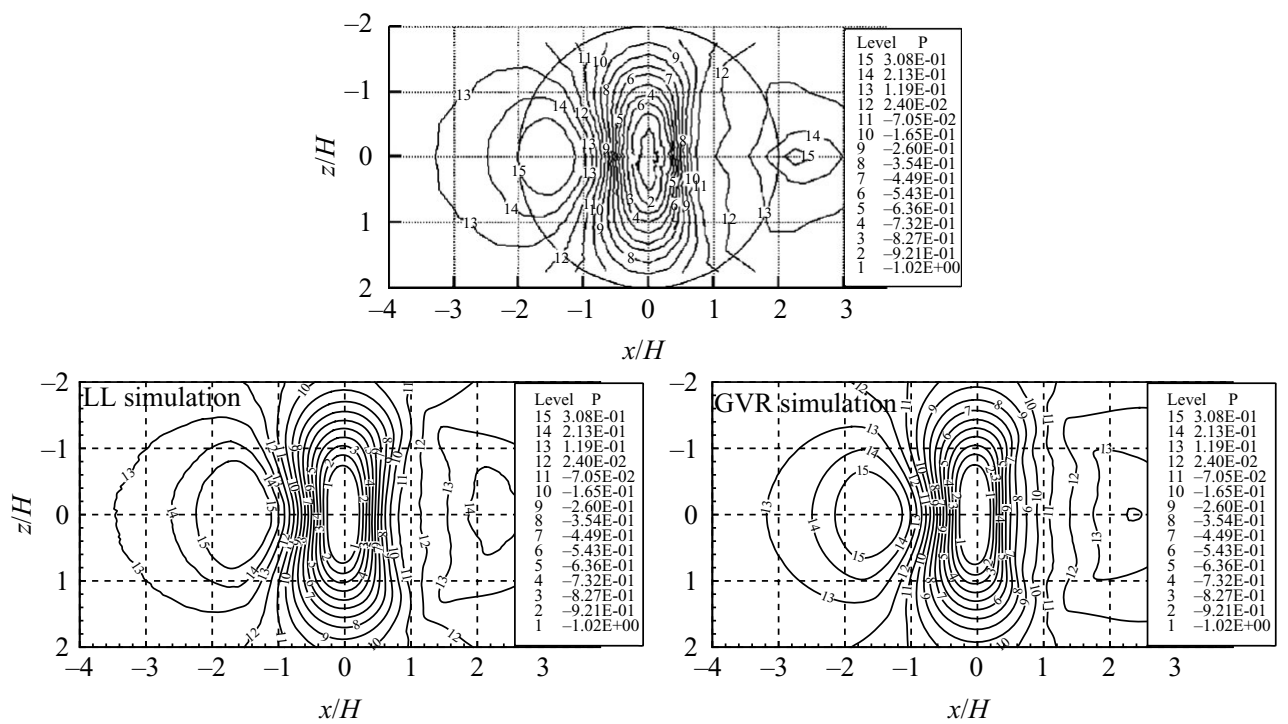

Figure 10. Contours of the pressure coefficient on the hill. Experimental data and corresponding subfigure are taken from Simpson et al. (2002).
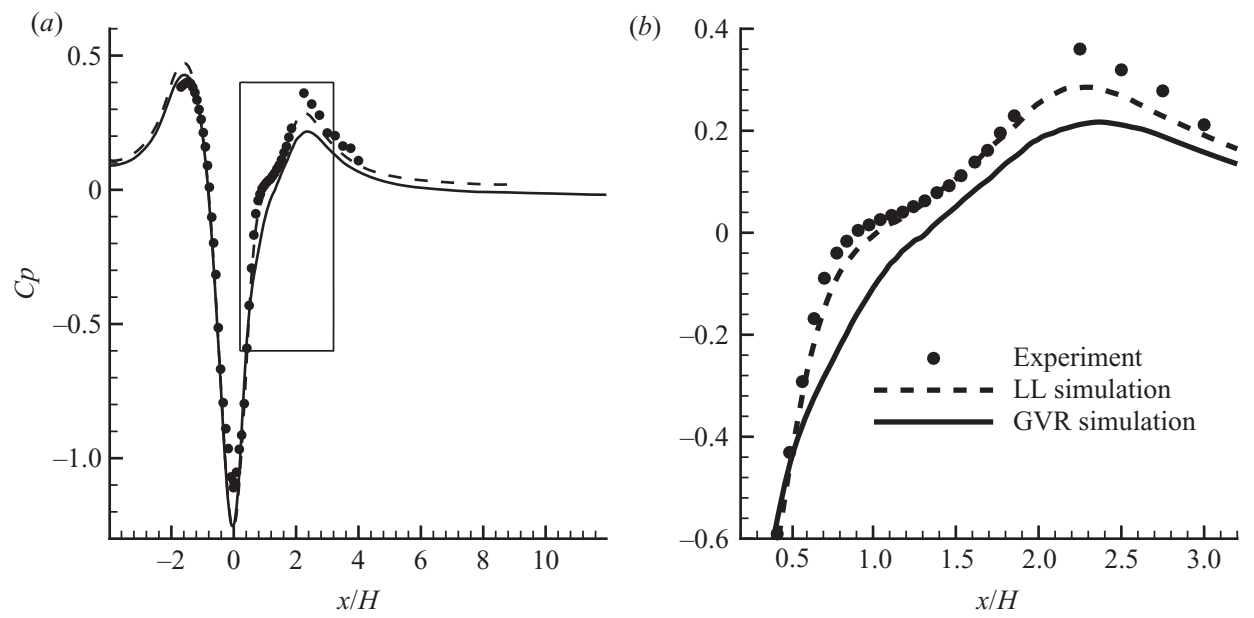

Figure 11. Pressure coefficient along the hill surface at the centre plane. (a) Full view; (b) zoomed-in view around the separation region. Experimental data are from Simpson et al. (2002).

Although the pressure is often a quantity that is well predicted, even when the near-wall velocity field is moderately erroneous, the present flow is an exception, in so far as the pressure is here an extremely sensitive indicator of the structure of the recirculation zone. As this zone is thin - the maximum wall-normal distance of the zero-velocity line within the recirculation zone is around $0.05 \mathrm{H}$ - even slight variations in the predicted velocity field within and around the recirculation zone can have a substantial impact on the pressure. Tessicini et al. (2007) show that failure to capture the separation zone, because of excessive grid coarseness, leads to the pressure plateau being missed altogether and thus to the pressure coefficient rising to 0.6 at 

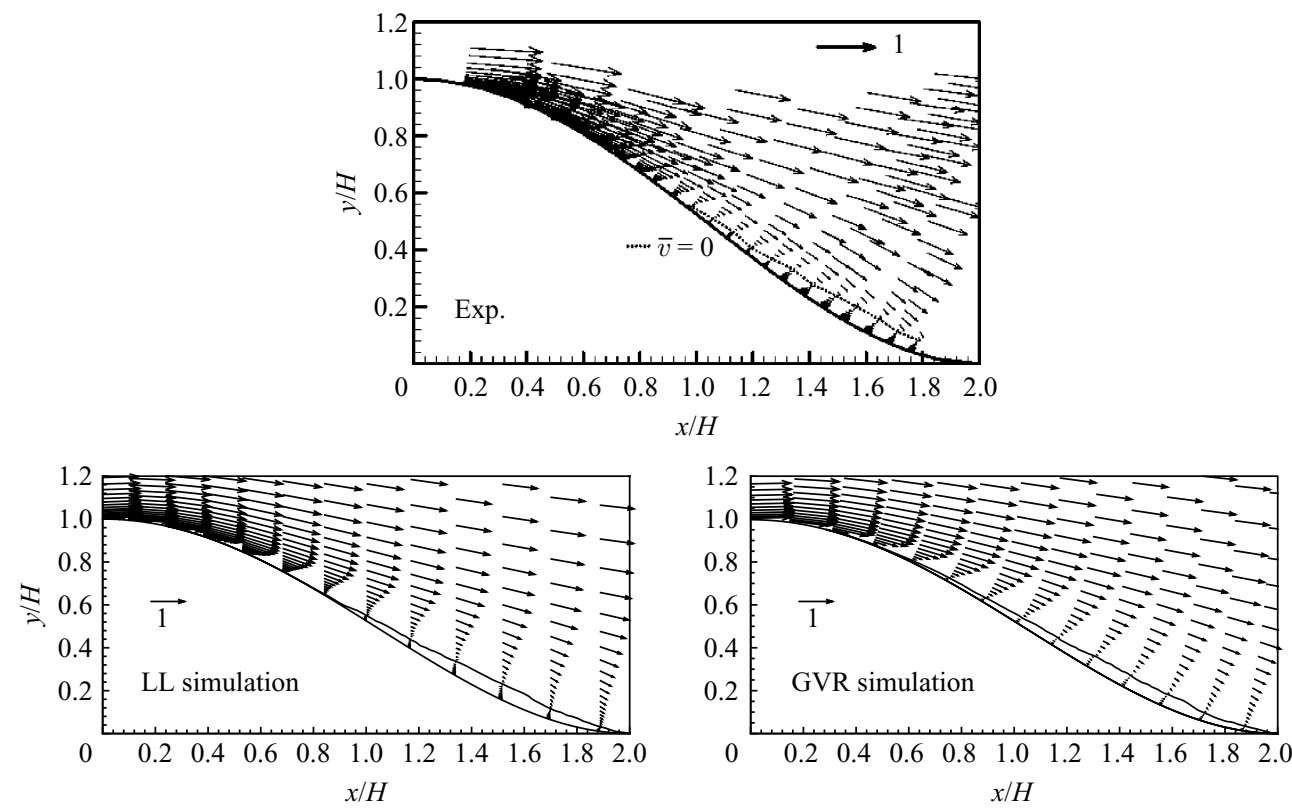

FIGURE 12. Velocity field across the centre plane in the leeward of the hill. The solid lines are the zero- $\langle u\rangle$-velocity lines, a good indication of the recirculation zone thickness. Experimental data and corresponding subfigure are taken from Byun \& Simpson (2006).

around $x / H=1.5$, i.e. in the middle of the plateau in figure 11 . The authors are of the view that resolution very close to the wall, especially in the leeward part of the hill, plays an important role in respect of the ability of the simulation to represent the pressure correctly. While GVR's streamwise and especially spanwise resolution is better than LL's, the wall-normal resolution very close to the wall is somewhat better in LL's simulation (figure $2 d$ ), and this may well be the origin of the differences in the predicted separation pattern and hence in the pressure coefficient.

\subsection{Topology and turbulence in the separation region}

Some comments on the flow structure in the separated region have already been made in the previous section, as far as this structure appertained to the discussion of the pressure field. Here, the flow properties within the separated region are considered in greater detail.

The predicted time-mean velocity field and contours of turbulence energy across the symmetry plane $z / H=0$ are presented in figures 12 and 13 , respectively, both in comparison with corresponding LDV measurements by Byun \& Simpson (2006). In the former figure, the thin lines close to the hill surface identify the locus $\langle u\rangle=0$. Both the experiment and the simulations show the reverse-flow region to be very thin. While the maximum thickness of the layer predicted by both simulations is in good agreement with the experimental value, there are clear differences in respect of the variation of the thickness, especially as the upstream separation node is approached. The experimental separation node is reported to be located at $x / H=0.96$, as derived from near-wall LDV measurements. Although the measurements show little variation in the position of the flow reversal within the range $11<y^{+}<41$, the accuracy of its location may well be subject to some uncertainty, because the recirculation zone tapers very gently towards the separation line, and this tends to accentuate the 

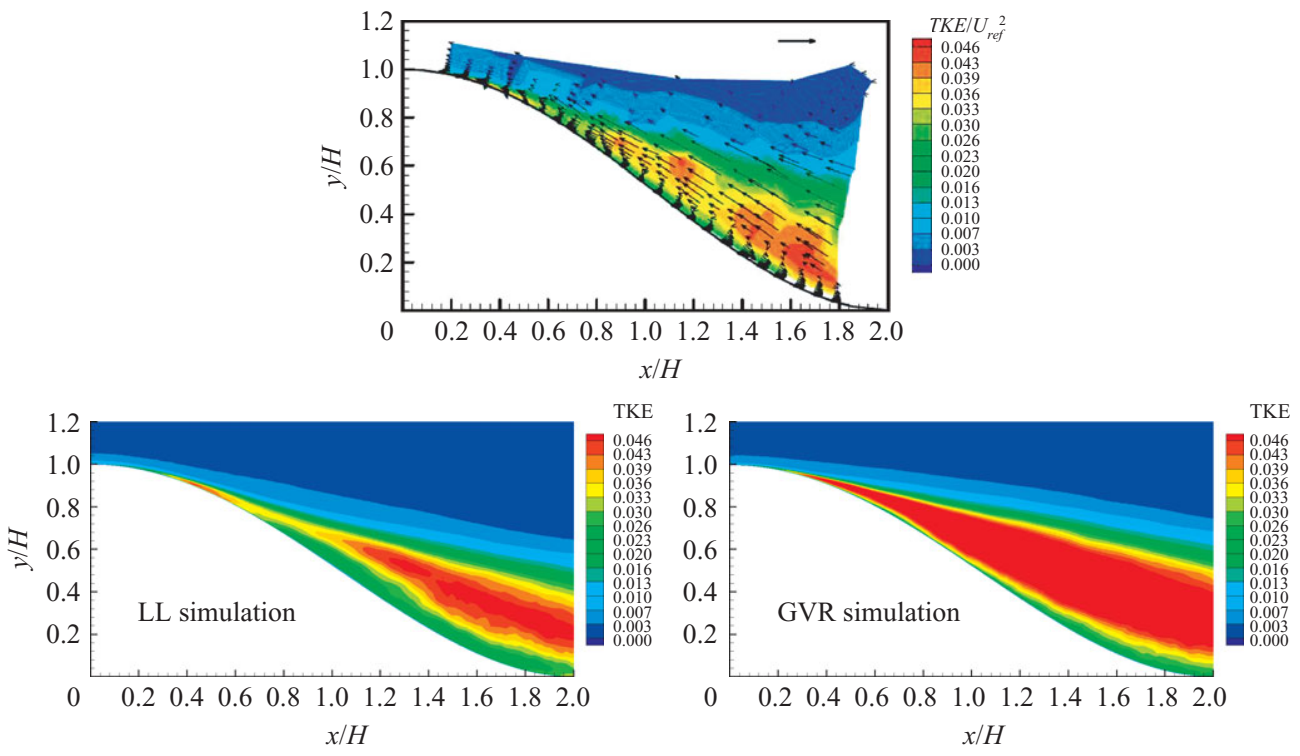

FiguRE 13. Turbulent kinetic energy distribution across the centre plane in the leeward of the hill. Experimental data and corresponding subfigure are taken from Byun \& Simpson (2006).

consequence of near-wall-resolution limitations. LL's separation point appears, on first sight, to be in good agreement with the experimental value, while GVR's location appears to be too close to the hill crest, at around $x / H \sim 0.5$. In fact, as shown later, by reference to topology maps, both simulations predict very similar separation locations at around $x / H \sim 0.3$, i.e. substantially upstream of the experimental value. The difference between the simulations thus lies in the rate at which the thickness of the recirculation zone diminishes to zero towards the hill crest, LL's recirculation zone being significantly thinner in the range $x / H=0.3-0.9$. These differences in thickness variations might appear rather unimportant, but they have, in fact, a major impact on the pressure field, as noted in the previous section. Both simulations predict the location of reattachment at around $x / H \sim 2$, closely matching the experimental location.

It is appropriate to point out here that the thinness of the recirculation zone makes its accurate capture, both experimentally and computationally, an extremely challenging task. It needs to be appreciated that the separation pattern varies substantially over time and space, to the extent that separation is intermittent, so that the time-mean properties of the separated zone are likely to be very sensitive to the turbulence dynamics of the entire flow, as well as to the precise manner in which the turbulent inlet conditions are prescribed. More generally, this layer is likely to be extremely sensitive to very small variations in both the computational or experimental conditions whatever their origin. As noted in the previous section, one possible contributor to the differences is the mesh density within the recirculation zone, especially the wall-normal density, which is somewhat higher in LL's simulation in the region $y / H<0.02$, as shown in figure $2(d)$.

Turbulence energy contours are shown in figure 13. One initially surprising aspect of the experimental observations is the presence of a patch of high turbulence energy just downstream of the hill crest, around $x / H \sim 0.3$, i.e. in a region well upstream of 
the recorded separation point $x / H \sim 0.9$. As noted earlier, the simulations suggest in contrast that the area in which a high level of $k$ occurs is around the separation line. Thus, the simulations bring to light the fact that the high-turbulence area is not in an attached region, but a consequence of the unsteady separation well upstream of the experimentally recorded position. The turbulence field of LL's simulation shows especially well the thin zone of high turbulence in the region $0.2<x / H<0.6$. It will be shown later that this region is also characterized by an especially high level of production of turbulence energy near the surface, consistent with the thin separated layer.

The high level of turbulence arising in the separated shear layer as it evolves beyond $x / H \sim 0.8$ is entirely in accord with expectation. This layer thickens progressively in the downstream direction, and with it, the region of high turbulence energy also becomes more extensive. The GVR simulation predicts larger values of $k$ than both the LDV measurements and the LL simulation, and this provides a further indication that the recirculation region returned by the former simulation is somewhat excessive. As shown in the next section dealing with the wake, the GVR simulation also predicts higher values of $k$ than the LL simulation in the wake region, well downstream of reattachment, but in that case agreement of the GVR predictions with hot-wire measurements (Ma \& Simpson 2005) of $k$, regarded as more accurate than the LDV data at that location, is close and better than that achieved by LL's simulation. This could indicate some inconsistencies between the two sets of measurements, but could also be attributed to the inferior resolution of the LL simulation due to the coarser grid it uses especially in the wake region.

The overall view conveyed by figures 12 and 13 is complemented by figure 14, which shows profiles of mean and r.m.s. velocities as a function of the wall-normal distance in wall units, in the symmetry plane. Attention is drawn to the fact that the friction velocity used for normalization is at its reference value that prevails upstream of the hill at $x / H=-4.0$. Here, the comparison is made with the LDV data reported by Byun (2005). To this end, the Cartesian components of the computed velocity field in the symmetry plane were projected onto the wall-tangential and wall-normal directions. Figure 14(a) shows the mean tangential velocity $\left\langle u_{t}\right\rangle$ just upstream, $x / H=0.18$, of the (computed) separation location. At this location, the flow is observed to have experienced strong acceleration by the hill, the flow speed at the edge of the boundary layer being roughly $30 \%$ higher than $U_{\text {ref }}$. Also, the boundary layer has become very thin at this location, its thickness being somewhat less than 100 wall units. This compares with a boundary-layer thickness of $\delta=0.5 \mathrm{H}$ upstream of the hill, which corresponds to approximately 2300 wall units. Figure $14(b)$ shows the same quantity downstream of separation, in the recirculation region, $x / H=1.31$. At this location, the recirculation region is well established in both simulations and in the experiment, and it is several hundred wall units thick. Figures $14(c-d)$ show profiles of the corresponding r.m.s. velocities in the wall-tangential and normal directions. Upstream of separation, the tangential fluctuations are significantly higher than the normal fluctuations, with the ratio being approximately 4 below $y^{+}=50$. At this location, high levels of fluctuations occur in the region below $y^{+}=100$, corresponding to the high strain rate observed in the associated velocity profile. In the recirculation region, the tangential fluctuations are roughly twice the normal fluctuations and are distributed across a broad layer extending to $y^{+} \sim 2000$. The peak is attained at $y^{+} \sim 1000$, which corresponds to the central part of the separated shear layer at this location. In general, both simulations present a reasonable agreement with the experimental data. The fluctuations predicted by GVR are somewhat higher than 

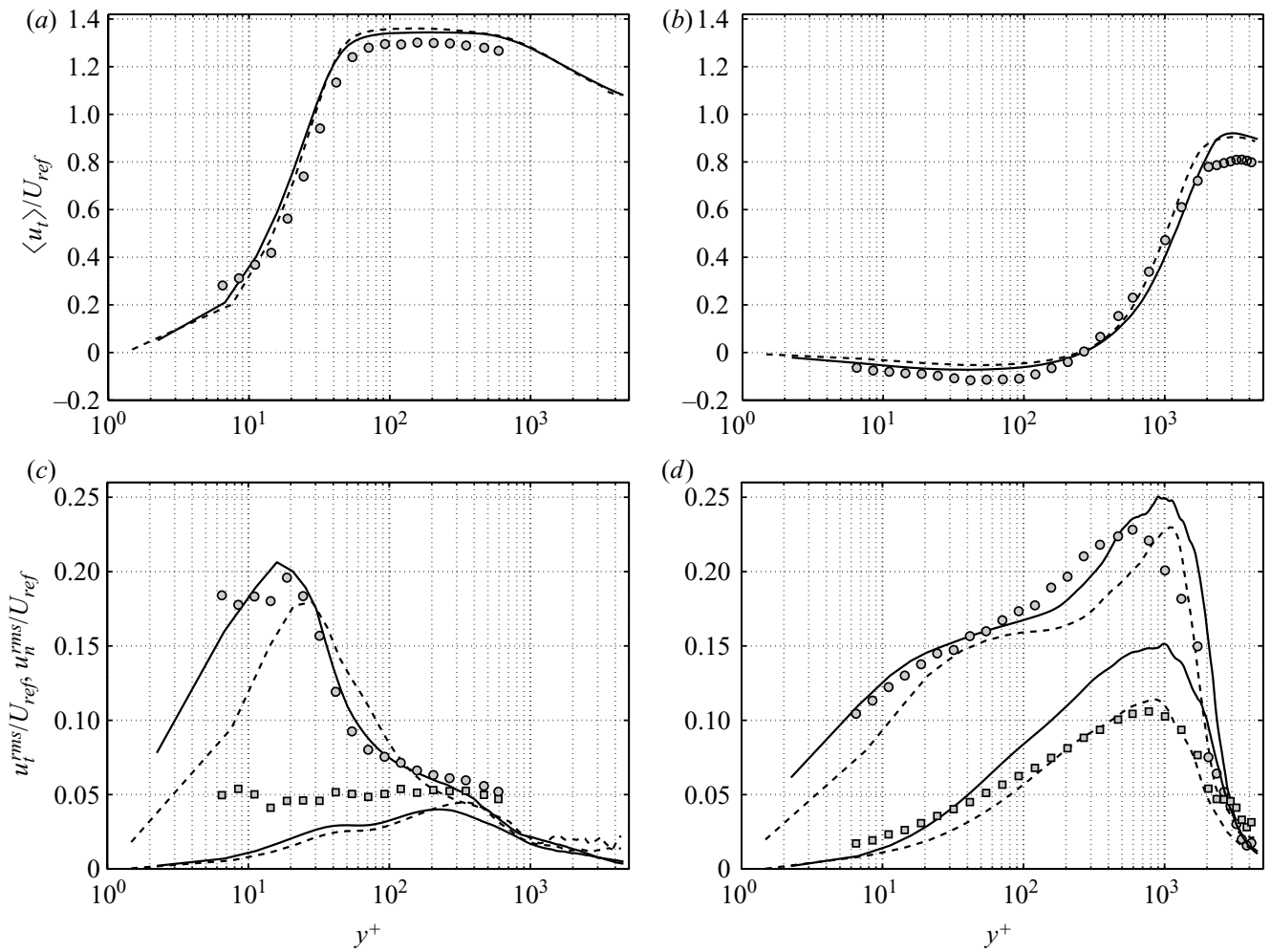

Figure 14. $(a-b)$ Mean tangential velocity $\left\langle u_{t}\right\rangle$ as a function of $y^{+} .(c-d)$ r.m.s. tangential velocity $u_{t}^{r m s}$ (upper curves) and r.m.s. normal velocity $u_{n}^{r m s}$ (lower curves) as a function of $y^{+} . z / H=0$. (a) and (c) Upstream of separation $x / H=0.18$. (b) and $(d)$ Downstream of separation $x / H=1.31$. Solid lines, GVR; dashed lines, LL; symbols and experimental data are from Byun (2005).

those predicted by LL, as expected from the contours of turbulent kinetic energy discussed above.

The previous discussion (on figures 12-14) restricted itself to the flow in the symmetry plane. A broader view of the three-dimensional structure of the mean flow is provided by streak-line maps across wall-parallel surfaces that cut through the separated zone at fixed wall-normal distances. Such maps projected onto the $x-z$ plane are shown in figures $15-17$ together with contours of turbulence energy. Figure 15 shows the results of LL, figure 16 shows the results of GVR and figure 17 plots the measurements of Byun \& Simpson (2006).

At the location closest to the wall, $y^{+} \sim 2$, the predicted flow is very similar in both simulations with separation occurring almost along the same locus $-x / H \sim 0.3$ at the centreline. Reference to figure 12 brings out the fact that the reverse-flow layer is extremely thin near the wall, especially in LL's simulation. Figure 17 suggests that the experimental separation line is well downstream of the computed line, but the uncertainties associated with this difference have already been noted. Farther away from the wall, the zone of flow reversal progressively moves downstream in both simulations, but at a faster rate in the LL simulation, consistent with the fact that the thickness of the reverse-flow layer predicted by this simulation is lower. One of the main differences between the experimental and computational topology maps relates 

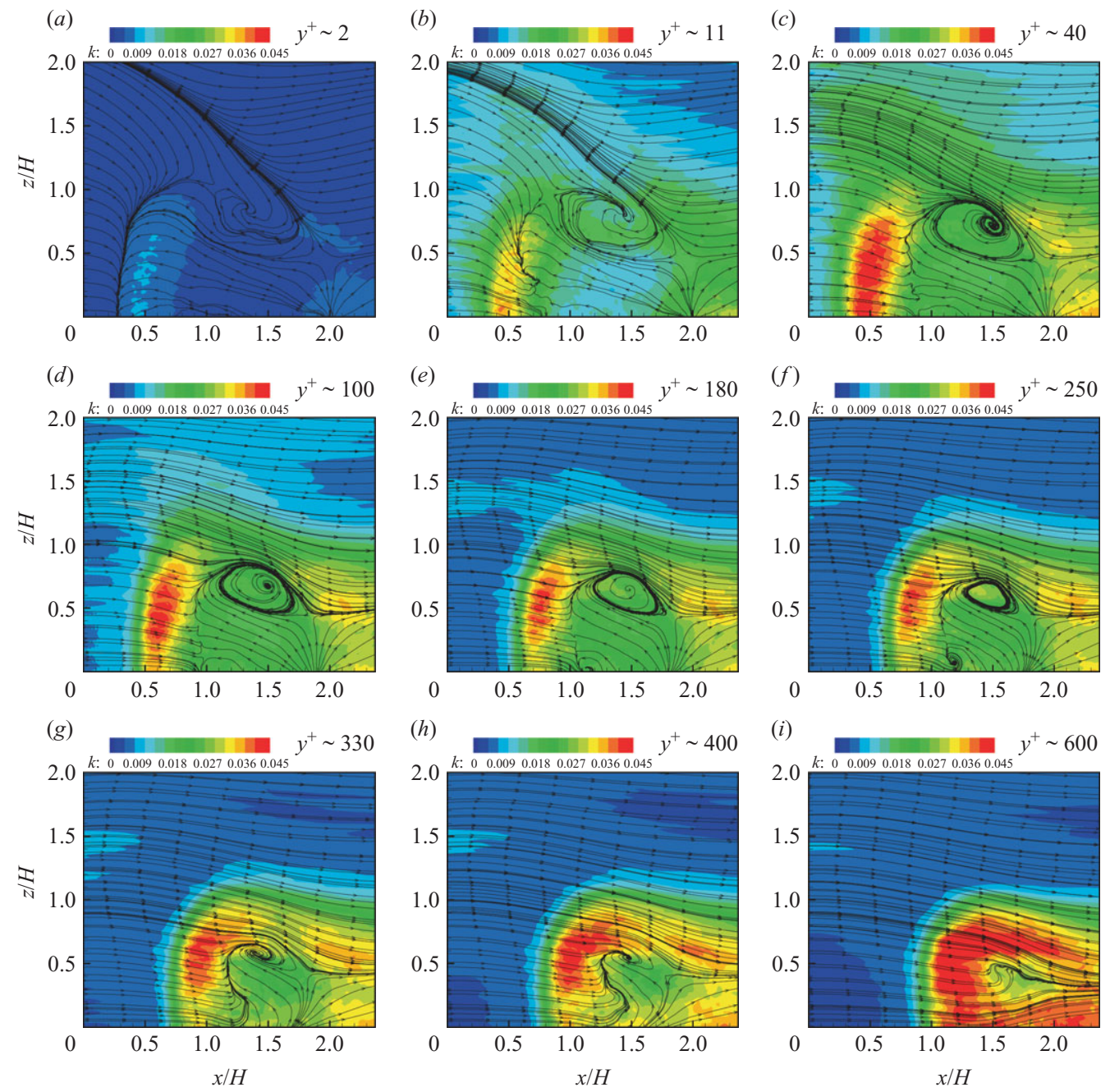

FIGURE 15. Flow topology predicted by LL simulation. Colour represents turbulent kinetic energy.

to the structural variation with height close to the wall. The experiments show hardly any change in the flow-reversal line in the layer $11<y^{+}<41$, while the simulations not only show the flow-reversal line to be well upstream of the experimental locus, but also indicate that the flow experiences significant wall-normal variations. In LL's simulation, the shift is somewhat larger, and this reflects the extreme thinness of the reverse-flow layer returned by this simulation in the upstream area. These differences add weight to the suggestion that resolution limitations in the experiment may well have prevented a proper representation of the near-wall flow and of the actual separation line. Beyond $y^{+} \sim 180$, flow reversal occurs broadly at the same position in both simulations.

Both simulations return very similar features for the outer vortex that emanates from the focal point seen in the topology map (figure $8 b$ ). Thus, at $y^{+} \sim 180$, the centre of this vortex is located roughly at $x / H=1.5, z / H=0.7$, somewhat farther downstream than in the experiment where the centre is at $x / H=1.4$. Significant 

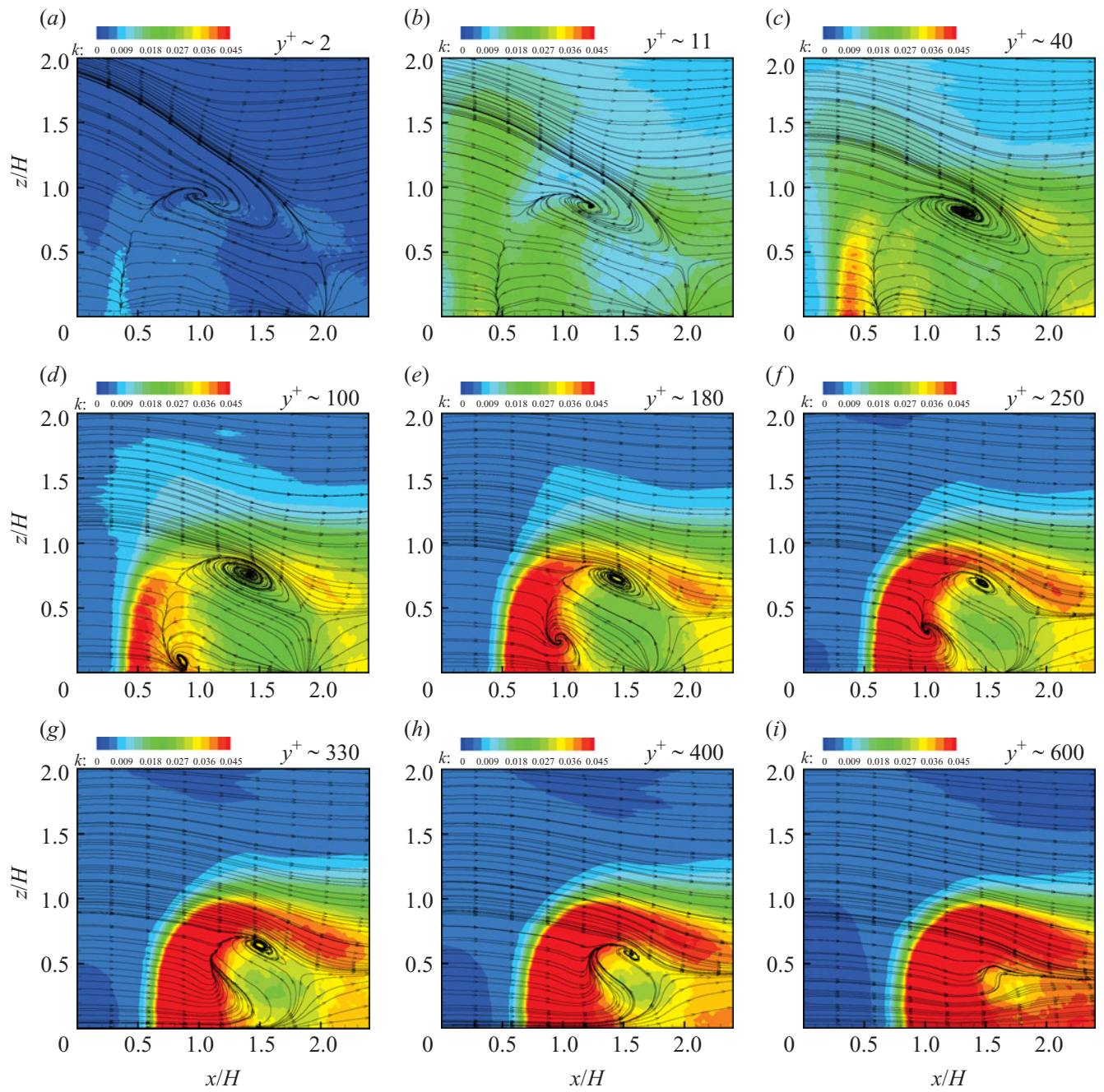

FIGURE 16. Flow topology predicted by GVR simulation. Colour represents turbulent kinetic energy.

differences arise, however, in respect of the secondary vortex close to the centreline. It is recalled that this vortex (or pair of vortices) evolves from the forward separation node, as a consequence of the transverse pressure gradient-inducing motion along the separation line towards the centre plane, between the two saddle points on that line (figure $8 b$ ). The vorticity accumulating at the separation node, from both sides of the centreline, has to be shed away from the wall. This process occurs in both simulations, and it is shown later that this vorticity is contained, in almost equal measure, in both predicted wakes. However, it is clear that the secondary vortex is more pronounced in GVR's simulation. This may be partly due to LL's lower resolution away from the wall, but it also seems to be linked, to a considerable extent, to the differences in the thickness of the reverse-flow layer and the rate at which it changes with streamwise distance. Initially, and in line with the slow increase in $C_{p}$ (figure 11b), GVR's results show a faster thickening of the reverse-flow region. Beyond $x / H \simeq 1$, the position reverses due to the penetration of forward-moving fluid into the reverse-flow region 

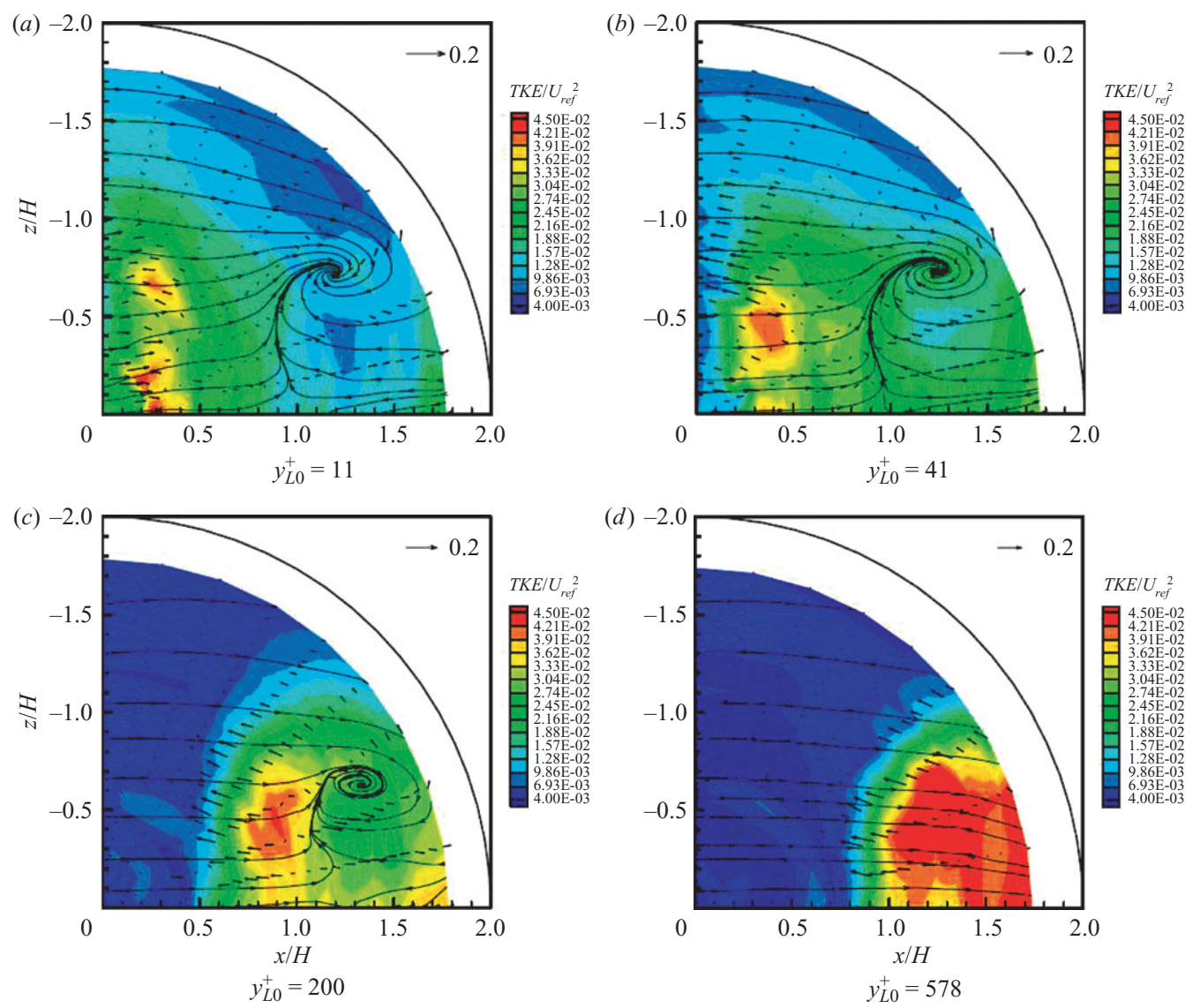

FIGURE 17. Flow topology (lines) and turbulent kinetic energy (colour) measured by Byun \& Simpson (2006).

associated with the formation of the secondary vortex. Thus, in this region, LL's reverse-flow layer thickens more rapidly and $C_{p}$ develops more of a plateau. Beyond $x / H \simeq 1.2-1.3$, both simulations return virtually the same thickness. Based on these observations, it is conjectured that the secondary vortical structures predicted by GVR's simulation are likely to be too large in size and intensity.

The contours of turbulent kinetic energy in figures 15-17, overlaid onto the flow maps, are consistent with the principal observation derived from figure 13, namely that GVR's simulation predicts higher values of $k$ than both LL's and the experimental levels, suggesting that the extent and intensity of the separation process predicted by GVR's simulation is at least slightly excessive. In general, high levels of $k$ are observed ahead and around the flow-reversal line, as well as on either side of the recirculation zone. The levels within the reverse-flow region are lower, reflecting the damping exerted by the wall on the upstream-flowing near-wall layer. At $y^{+}=40$, there is a spanwise patch of elevated $k$ at around $x / H=0.3-0.4$, which is observed in both simulations and the experiment. In both simulations, this patch is located just upstream of the flow-reversal line, while in the experiment, this line is located much farther downstream at around $x / H \sim 0.9$. The patch may be interpreted to reflect the highly unsteady nature of the separation process, in which the stagnation line at $y^{+} \sim 11$ extends, at times, well upstream of its mean location $x / H \sim 0.5$. The focus 


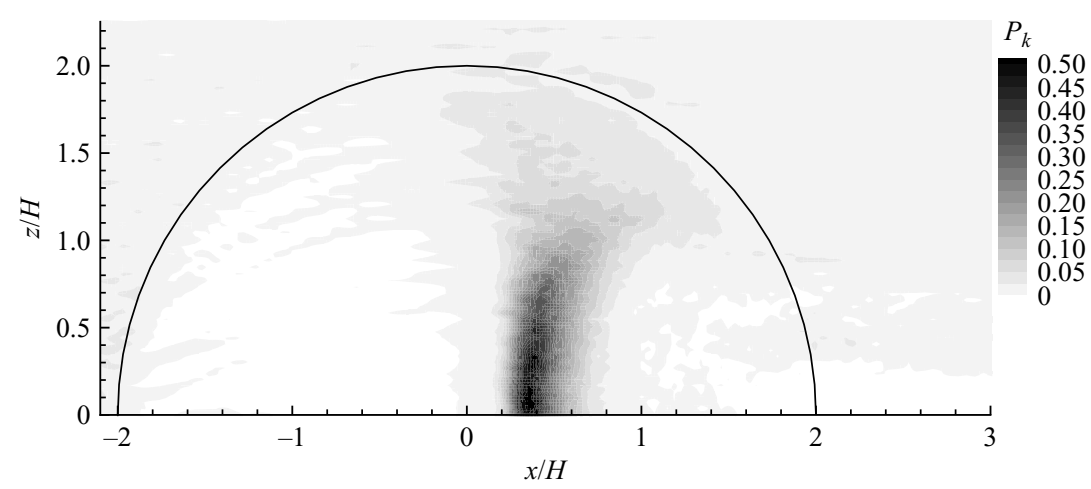

FIGURE 18. Contour of TKE production term across $y^{+}=40$ from LL.

on this $y^{+}$value is appropriate because it is at this height that turbulence is expected to rise quickly in the separated shear layer. Thus, in the simulations the location of the patch of elevated turbulence is consistent with the flow map. On the other hand, no such consistency arises in the case of the experiment, and this strengthens the interpretation that the experimental flow map at $y^{+}=11$ does not truly reflect the actual separation process at the hill surface.

A final piece of evidence supporting the above argument on the turbulence-energy fields is given in figure 18. This shows contours of the turbulence-energy production across a layer at around $y^{+}=40$. This plot arises from budgets extracted from LL's simulation. Wall-normal profiles of the terms contributing to the turbulence energy, not included herein, show the production to be mainly balanced by dissipation, with turbulent transport and pressure diffusion making significantly smaller contributions. The important feature in figure 18 is the high level of production predicted around $x / H=0.3$, consistent with the high near-wall turbulence energy at the same location in both the simulations and the experiment.

\subsection{Near wake}

Experimental data at the streamwise locations $x / H=2.62,3.63$ and 6.59 offer the opportunity to investigate the structure of the near wake in which the flow recovers from reattachment at $x / H=2$. Within this range, the flow is characterized by a combination of streamwise acceleration associated with the wake-recovery process, and transverse circulation with peak secondary velocity of around $15 \%$ associated with the streamwise realignment of the boundary-layer vorticity originating upstream of the hill and additional vorticity shed from the hill.

As pointed out in the previous section, there are two sets of measurements available for the near-wake flow - one obtained by means of LDV and the other with HWA. Of these, the latter are regarded (by the originators of the data, Ma \& Simpson 2005) to be more reliable than the former because of seeding problems in the LDV measurements. The HWA data are therefore used as a basis of the present comparisons, restricted to $x / H=3.63$. The phenomena observed in the other two streamwise locations for which data are available are very similar, as is the level of agreement between the simulations and the measurements.

Prior to a comparison and discussion of velocity and Reynolds stress profiles, it is instructive to present first an overall view of the streamwise evolution of the transverse motion in the wake. This is done in two parts: first, a series of predicted contour plots of mean streamwise vorticity are given in figure 19. These contours, covering 
(a)

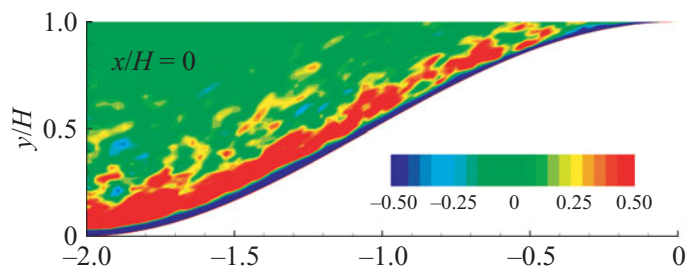

(b)

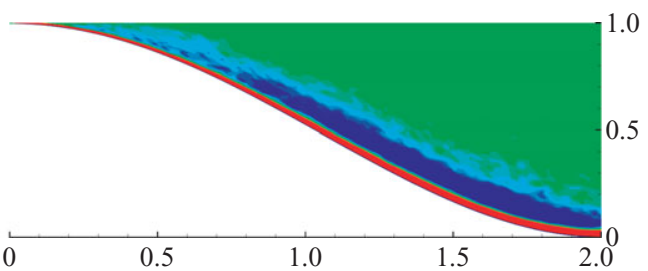

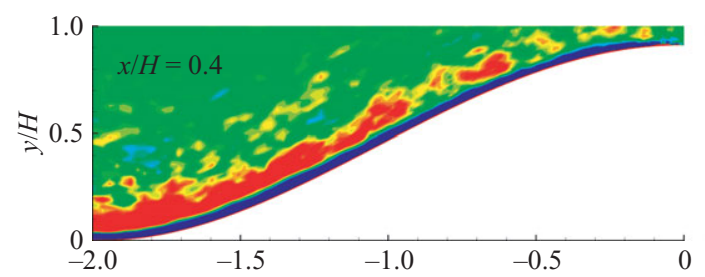
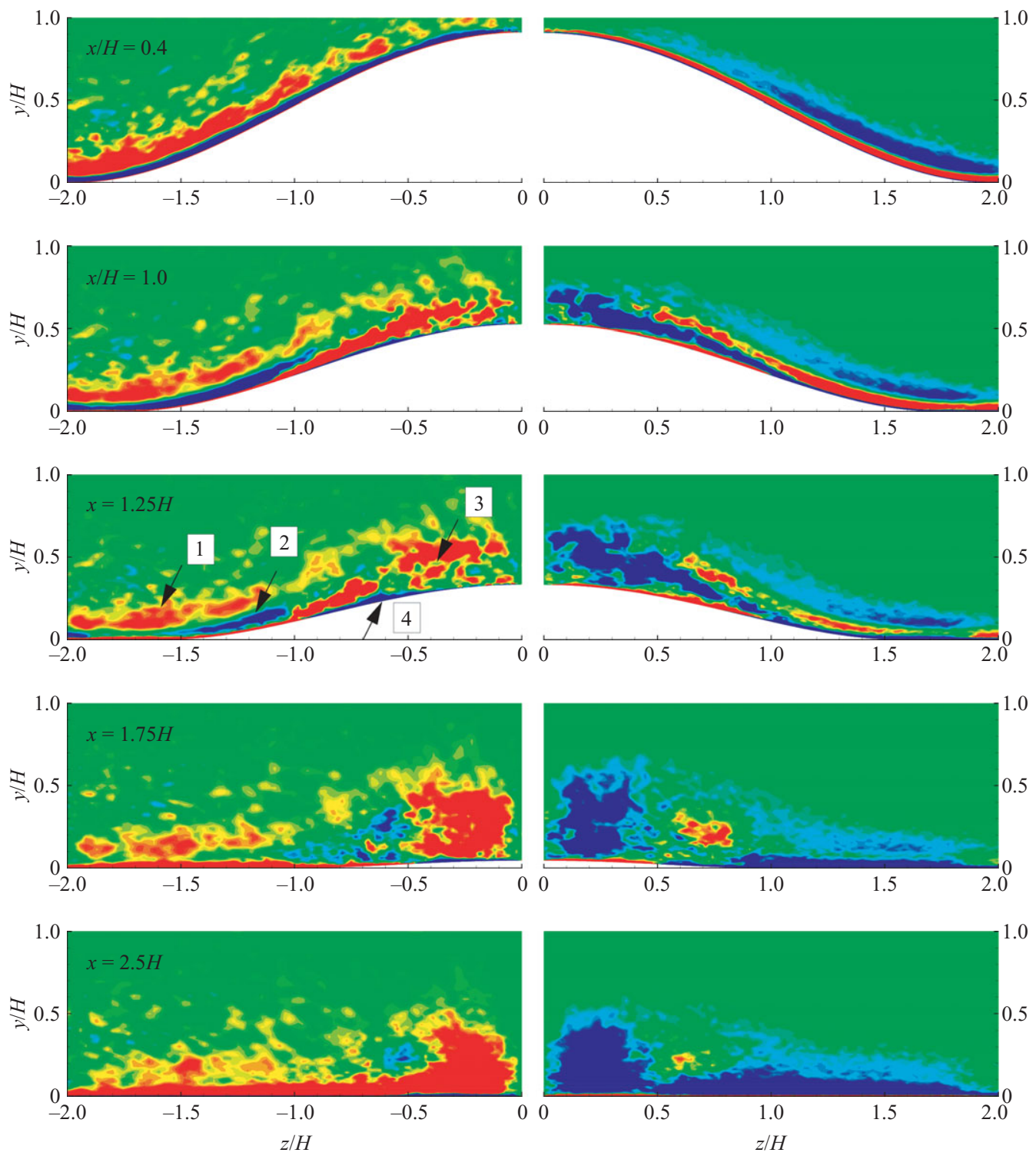

FIGURE 19. Mean streamwise vorticity at several transverse planes. (a) LL; $(b)$ GVR. Note that the streamwise vorticity field is antisymmetric with respect to the symmetry plane. 
(a)

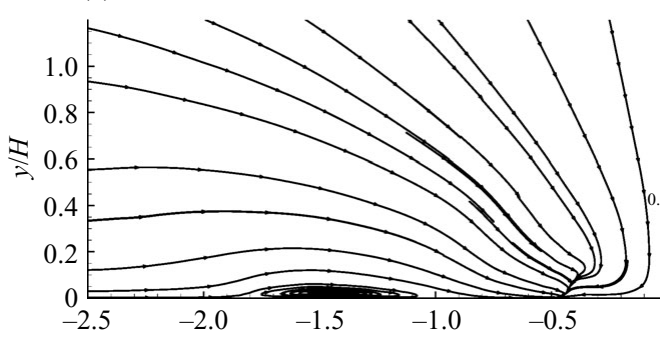

(b)
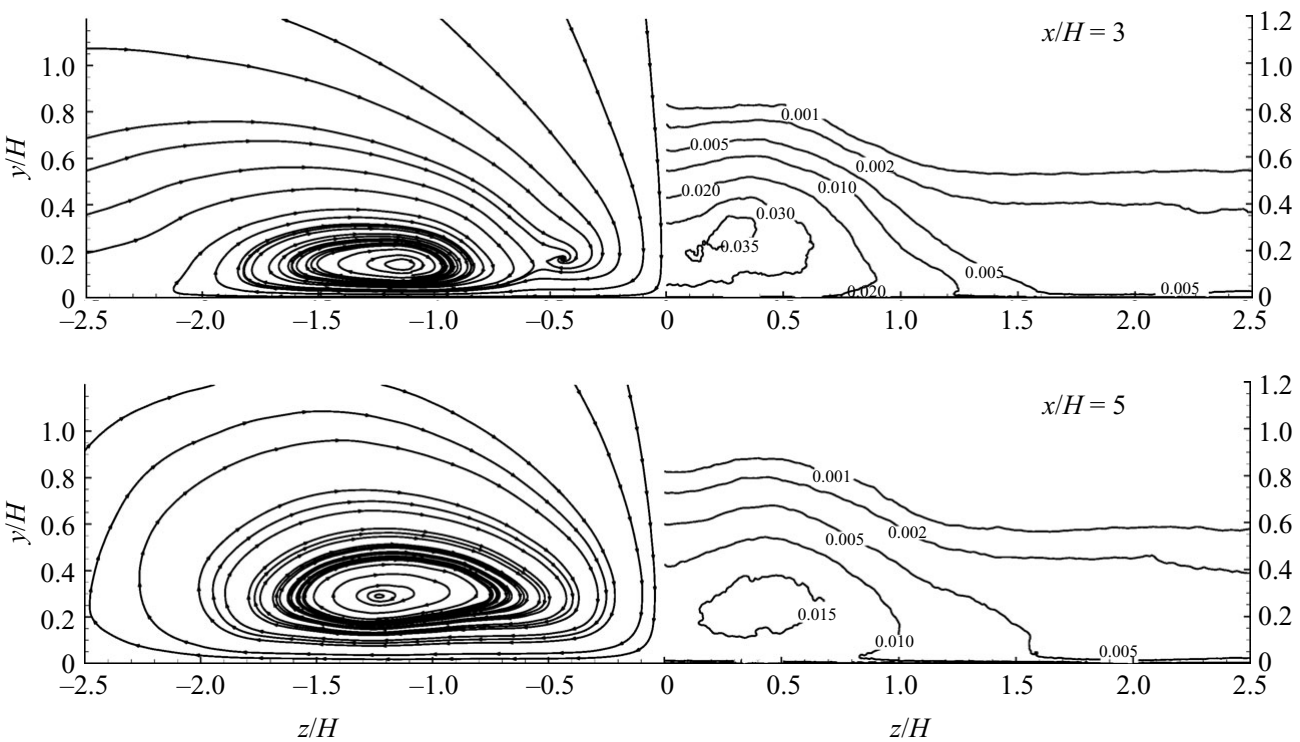

FIgURE 20. (a) Secondary motions at $x / H=2,3$ and 5. (b) Turbulent kinetic energy. Simulation GVR.

the streamwise stretch $0<x / H<2.5$, allow the formation of the wake structure to be traced to specific processes occurring in the leeward portion of the hill, specifically to features discussed earlier by reference to figures 15-17. Second, figure 20 shows the evolution of the transverse motion and turbulence energy farther downstream, covering the streamwise stretch $2<x / H<5$. Figure 19 is a composite of results arising from both simulations: LL's in figure 19a, and GVR's in figure $19 b$ (the colour reversal across the centreline reflects the change in the sign of the vorticity). Both sets show a remarkable degree of similarity. Most informative is the evolution of the flow beyond $x / H=1.0$. With attention focused on the left-hand side set of plots (figure 19a), four distinct regions may be recognized, identified numerically in the plot for $x=1.25 \mathrm{H}$ : an outer red region, 1 , of positive vorticity lying at some distance above the wall; an outer blue region, 2, of negative vorticity close to the wall; an inner red region, 3 , of positive vorticity above the wall; and a very thin blue inner layer, 4, of negative vorticity very close to the wall. The origin of the four regions are, respectively, as follows: 1, the vorticity of the boundary layer upstream of the hill, having been reoriented towards the streamwise direction; 2, the vorticity shed from the primary focal point in the separation zone, 3, the vorticity shed from the separation node, reflected by the secondary spiral close to the centre plane; and 4 , the vorticity in the very thin separated zone. 
As the flow progresses downstream, the inner red region, 3, associated with the secondary spiral, increases in size and overwhelms the vorticity that is associated with the primary focus in the topology map, 2. At $x=2.5 H$, the field is dominated by positive vorticity, reflecting a merger of the vorticity originating from the upstream boundary layer with that shed from the separation node.

The process described above is also reflected in figure 20. At $x / H=2$ and 3 , the inner vortical feature is associated with the inner red region of figure 19, while the inflexions in the contours of the secondary motion at $x / H=2$ in figure 20 are associated with the increasingly minor blue region at $x / H=1.75$ and 2.5 in figure 19, which originates from the separation focus. Eventually, the transverse motion is dominated by a single vortex assimilating the boundary-layer vorticity and the vorticity shed from the stagnation node. Thus, remarkably, the vorticity shed from the separation focus is relatively weak and eventually becomes unrecognizable due to diffusion and the dominance of the opposite vorticity.

An important effect of the secondary motion on the recovery in the wake is through advective transport of fluid having low streamwise momentum from the region downstream of the reattachment and the wall into the outer and upper parts of the wake, thus depressing the turbulence-induced recovery of the streamwise velocity. Clearly, therefore, the intensity of the transverse motion is expected to have a significant impact on the streamwise-velocity profiles, as brought out below by reference to the velocity profiles in the wake.

As regards the evolution of the turbulence energy in the wake, one informative observation is the rapid rate of diminution in the maximum level of energy, suggesting a strong contribution of convection and dissipation, relative to weak generation. This is confirmed in figure 21 which shows four contributions to the turbulence-energy budget across $x / H=3.63$ (the contribution of pressure diffusion is negligible). While the contours are rather 'noisy' - reflecting the need for extremely long integration times in the case of separated flows - the budget confirms the dominance of convection and dissipation with the gain by production being low, as is typical for wakes. Another interesting feature is the distortion of the turbulence contours by the convective action of the transverse motion, leading to a broadening region of high turbulence energy near the wall and a depression of the energy in the centre region. The consequence is a deformation of the contour lines into a kidney shape characteristic of strong convective motion by a vortex. Finally, it is remarked that the maximum turbulence energy is observed in the inner region in which the fluid originates from the separation zone, specifically from the fluid ejected from the vicinity of the separation node.

Wall-normal profiles of the mean streamwise $\langle u\rangle$ and spanwise $\langle w\rangle$ velocity, turbulence energy $k$ and the Reynolds shear stress $\left\langle u^{\prime} v^{\prime}\right\rangle$ are shown in figures 22 and 23, respectively, all at $x / H=3.63$. Each pair of plots forming a row corresponds to a particular spanwise location, namely (from top to bottom) $z / H=0,0.32,0.65$, 1.10 and 1.79. Measurements were performed on both sides of the symmetry plane, and both sets of data are included together with the corresponding computational solutions. This provides an indication of the experimental uncertainties.

The profiles in figure 22 contain features that are consistent with the interpretations derived from the global views discussed above. Thus, for example, the combination of spanwise inward motion at the outer $y / H$ region, combined with the spanwise outer motion at the wall, leads to the pronounced inflexions observed in the $\langle u\rangle$ profiles at $z / H=0.32$ and 0.65 . The profiles predicted by both simulations agree well with the measurements, in general, except for the excessive near-wall levels of $\langle u\rangle$ at the outermost $z / H$ locations returned mainly by LL's simulation. 

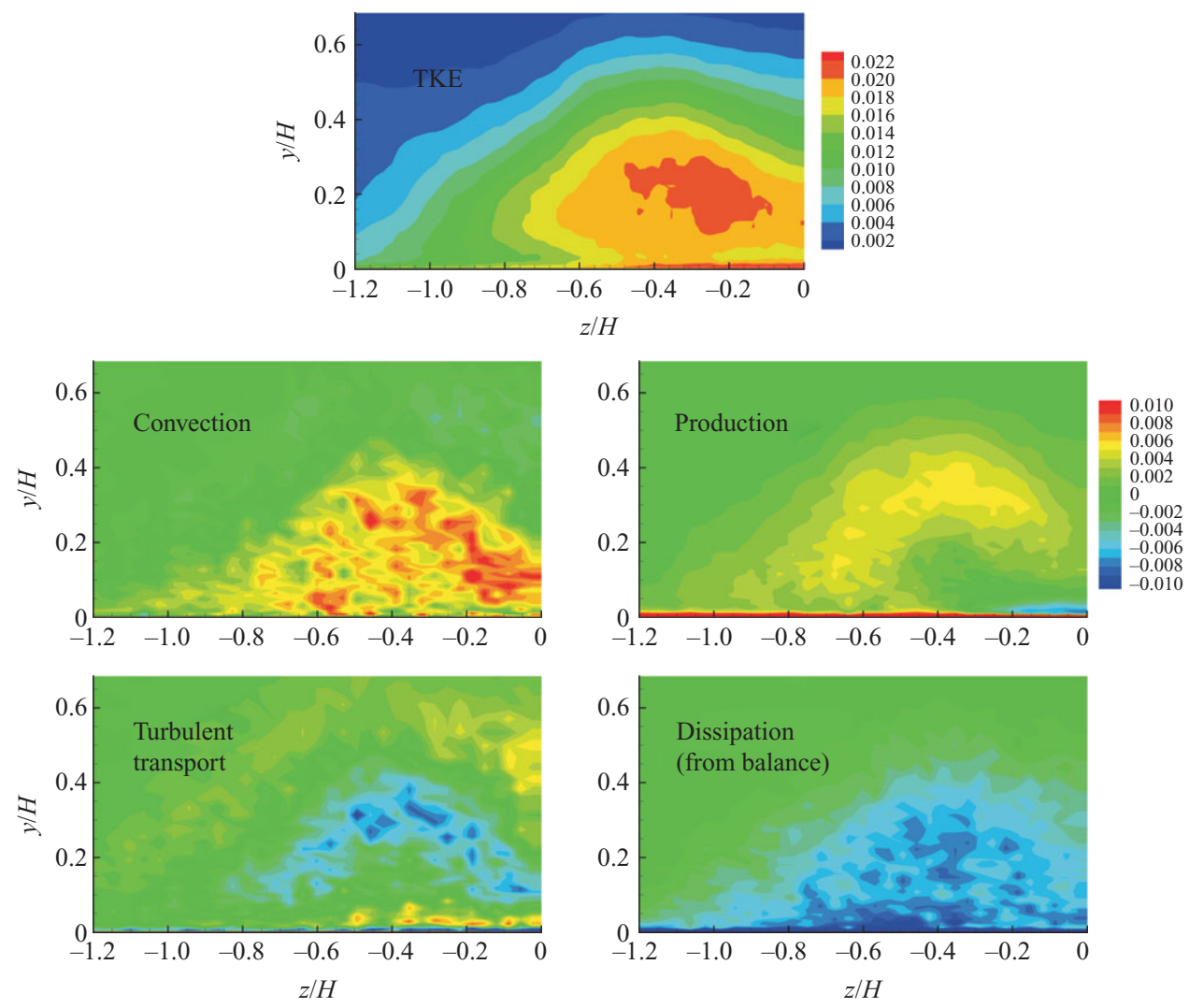

FIGURE 21. Contour of TKE budget in wake plane $x / H=3.63$ from LL.

Profiles for $k$ and $\left\langle u^{\prime} v^{\prime}\right\rangle$ are shown in figure 23. As noted earlier, when discussing the structure of the separation region, LL's simulation generally predicts lower levels of $k$ than GVR's simulation. While this was in accord with the experimental observations in the recirculation zone, better agreement is achieved in the wake by GVR's simulation, which is very good, overall. The decrease of the level of $k$ and $\left\langle u^{\prime} v^{\prime}\right\rangle$ with increasing spanwise position is well captured by both simulations. Attention is drawn to the fact that the maximum level of $k$ does not occur in the symmetry plane but around $z / H=0.32$. Reference to figure 20 shows, consistently, that the maximum turbulence energy at $x / H=3.0$ indeed lies at around $z / H=0.3$, and this is probably due to transport of highly turbulent fluid from the separated region into this part of the wake, suggested by the streaklines identifying the transverse motion. The somewhat too low level of turbulence activity returned by LL's simulation and certain defects in the $\langle u\rangle$ profile such as the near-wall bulge are likely to be due to the substantially lower resolution provided by LL's coarser grid in the wake, especially in outer spanwise regions.

\section{Analysis of the instantaneous flow and spectra}

\subsection{Instantaneous flow}

Any turbulent separated flow features high-intensity, highly unsteady, large-scale motions that render the instantaneous state drastically different from its time-averaged 
(a)
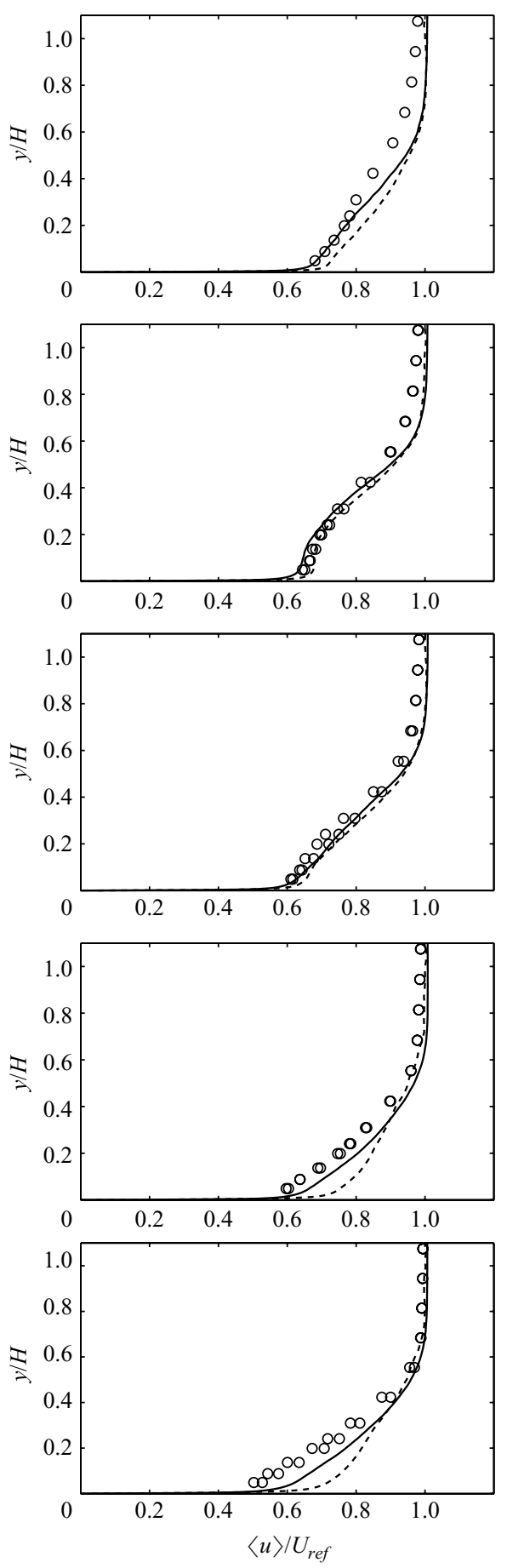

(b)
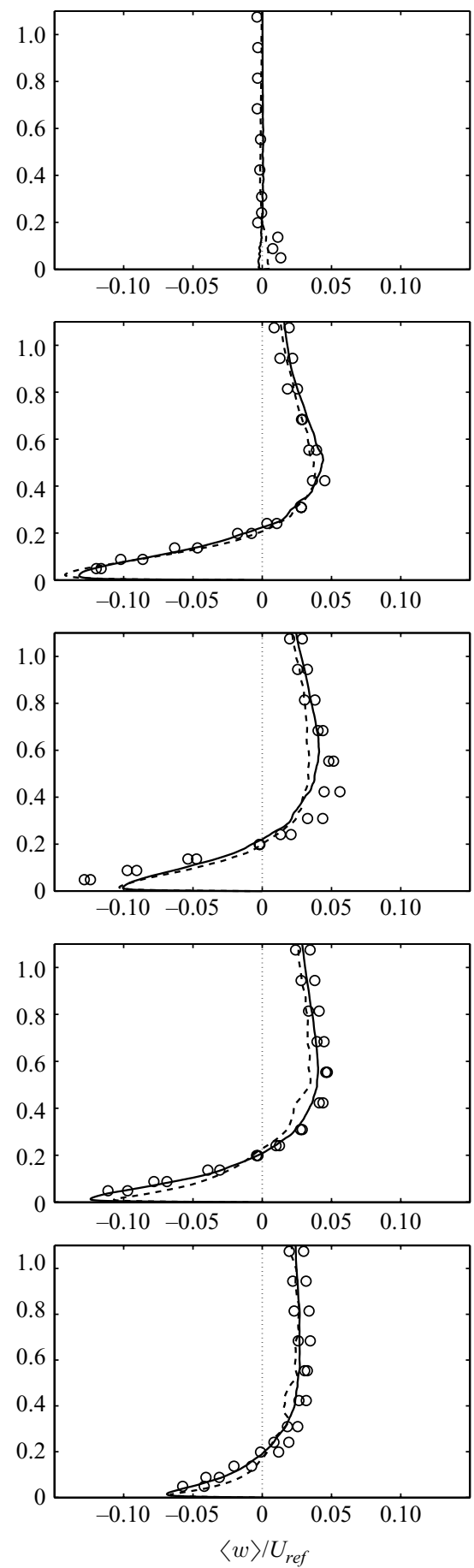

FIgURE 22. Profiles of $(a)$ streamwise $\langle u\rangle$ and $(b)$ spanwise mean velocity $\langle w\rangle$ at $x / H=3.63$. From top to bottom, $z / H=0,0.32,0.65,1.10$ and 1.79. Symbols, experiments; solid line, GVR; dashed line, LL. Experimental data are from Ma \& Simpson (2005). 
(a)
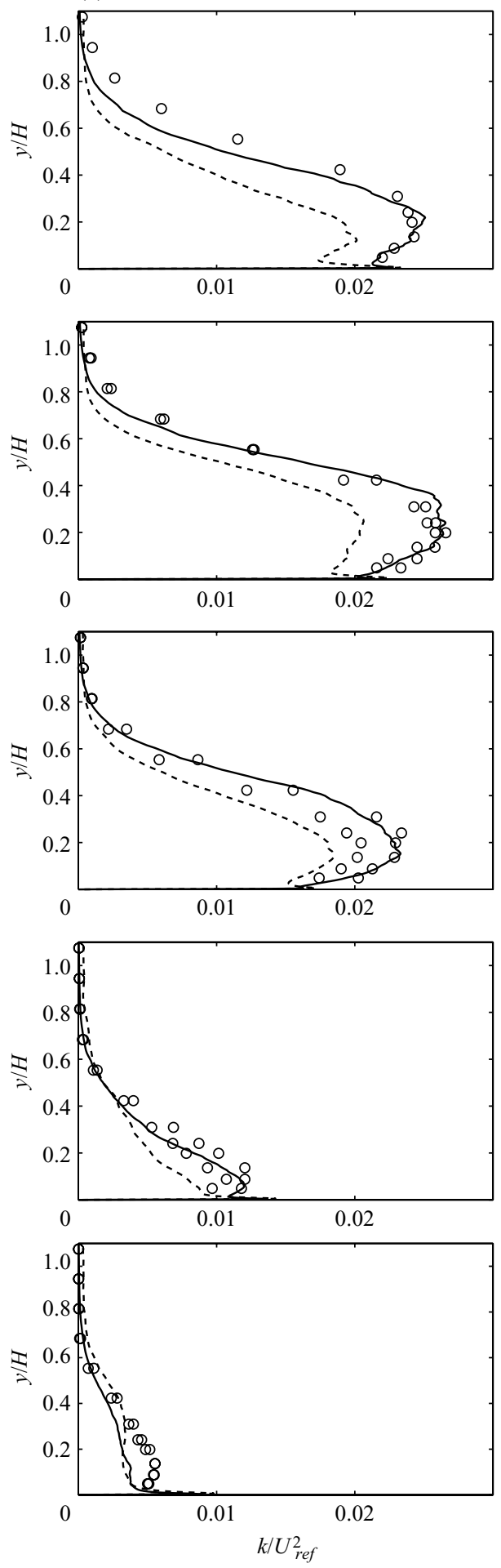

(b)
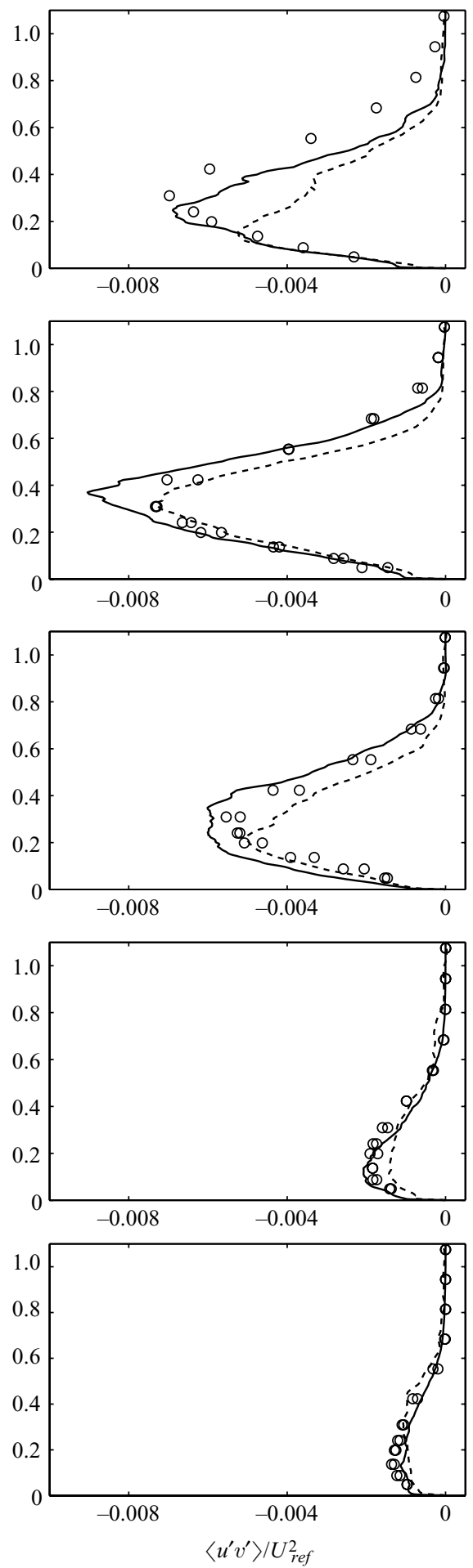

FiguRE 23. Profiles of $(a)$ turbulent kinetic energy $k$ and $(b)$ Reynolds shear stress $\left\langle u^{\prime} v^{\prime}\right\rangle$ at $x / H=3.63$. Here, $z$-positions and styles are as in figure 22. Experimental data are from Ma \& Simpson (2005). 

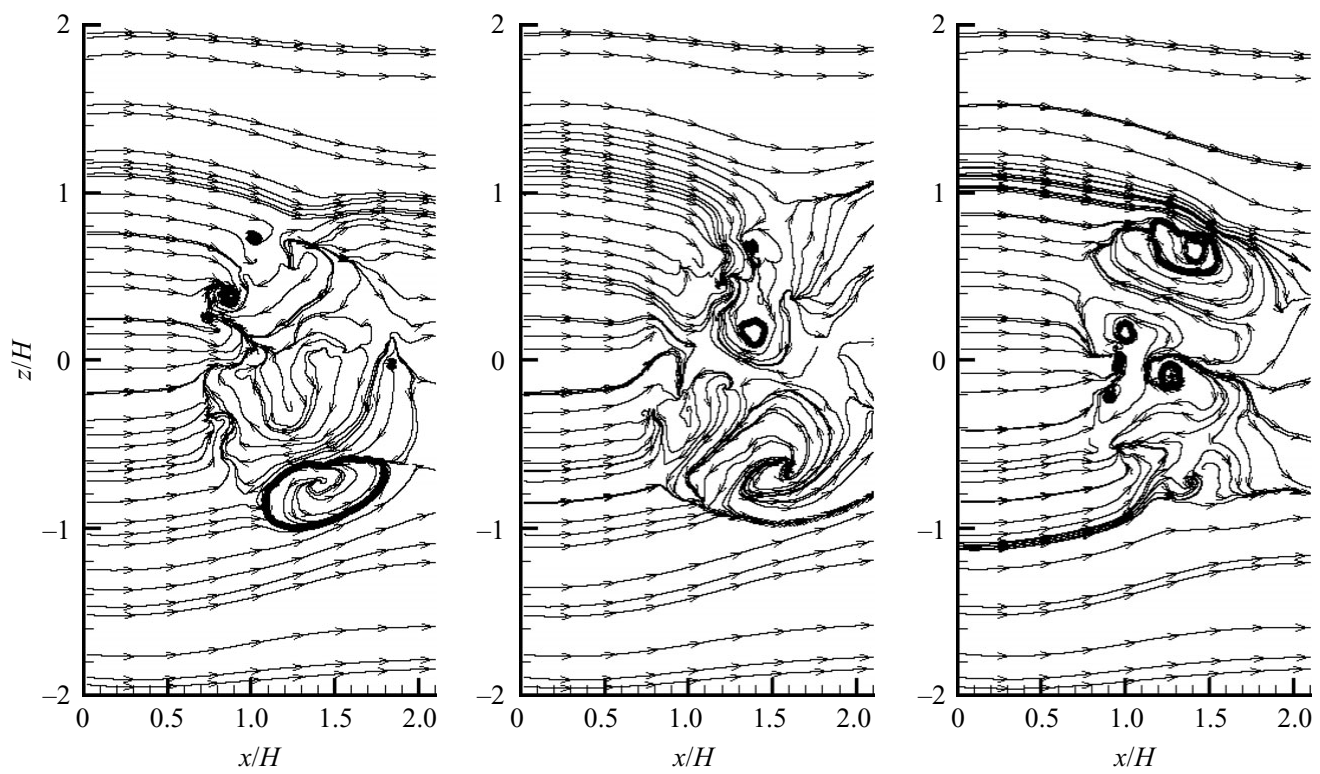

FIGURE 24. Visualization of the instantaneous flow in the backflow region in a grid surface close to the wall at three randomly selected instants from GVR.

counterpart. This is also the case with the present flow. A graphic illustration of this fact is given in figure 24, which shows three instantaneous topology maps selected randomly, but separated by at least $20 \mathrm{H} / U_{\text {ref }}$ each. When compared to the topology map in figure 8 , these images convey the complex multiplicity of time-varying focal point and nodes on the surface, images which bear no relationship to the timeaveraged result. More insight and understanding of the unsteady behaviour may be obtained by examining animations of the time-evolving topology at various wall distances. Closest to the wall they show that the separation is patchy and highly unsteady. The animations further show that dominant foci tend to migrate at irregular intervals from one side of the flow to the other - as suggested by the first and third images in figure 24 - and that the inner vortices form intermittently by outer fluid penetrating into the separation region and rolling up. Intriguingly, both the experiment and the simulations suggest the presence of long time-scale 'meandering' of the flow from one side of the hill to the other, and this is a subject discussed later by reference to spectra. However, it would be inappropriate to associate this phenomenon with conventional vortex shedding because of the much longer time scale in the present case, but there are strong indications of periodic events - although there is no obvious causality between this periodicity and the type of asymmetry conveyed in figure 24 .

Efforts to identify organized unsteady processes and structures can rest on the application of several visualization techniques to the time-evolving fields. Isosurfaces of velocity, vorticity and pressure may provide valuable clues. The visualization of isosurfaces of the so-called $Q$ and $\lambda_{2}$ criteria is another popular approach. Finally, POD is increasingly used to highlight the role of energetic low-order modes associated with periodic events. This last method has been attempted in the present case, but turned out to be much too costly to obtain converged fields for even the first three POD modes of the streamwise velocity, which were found in any event to carry only around $3 \%$ of the total energy of the flow (using 700 velocity fields covering 200 time units). For a flow of similar complexity to the present one, Manhart (1998) needed 

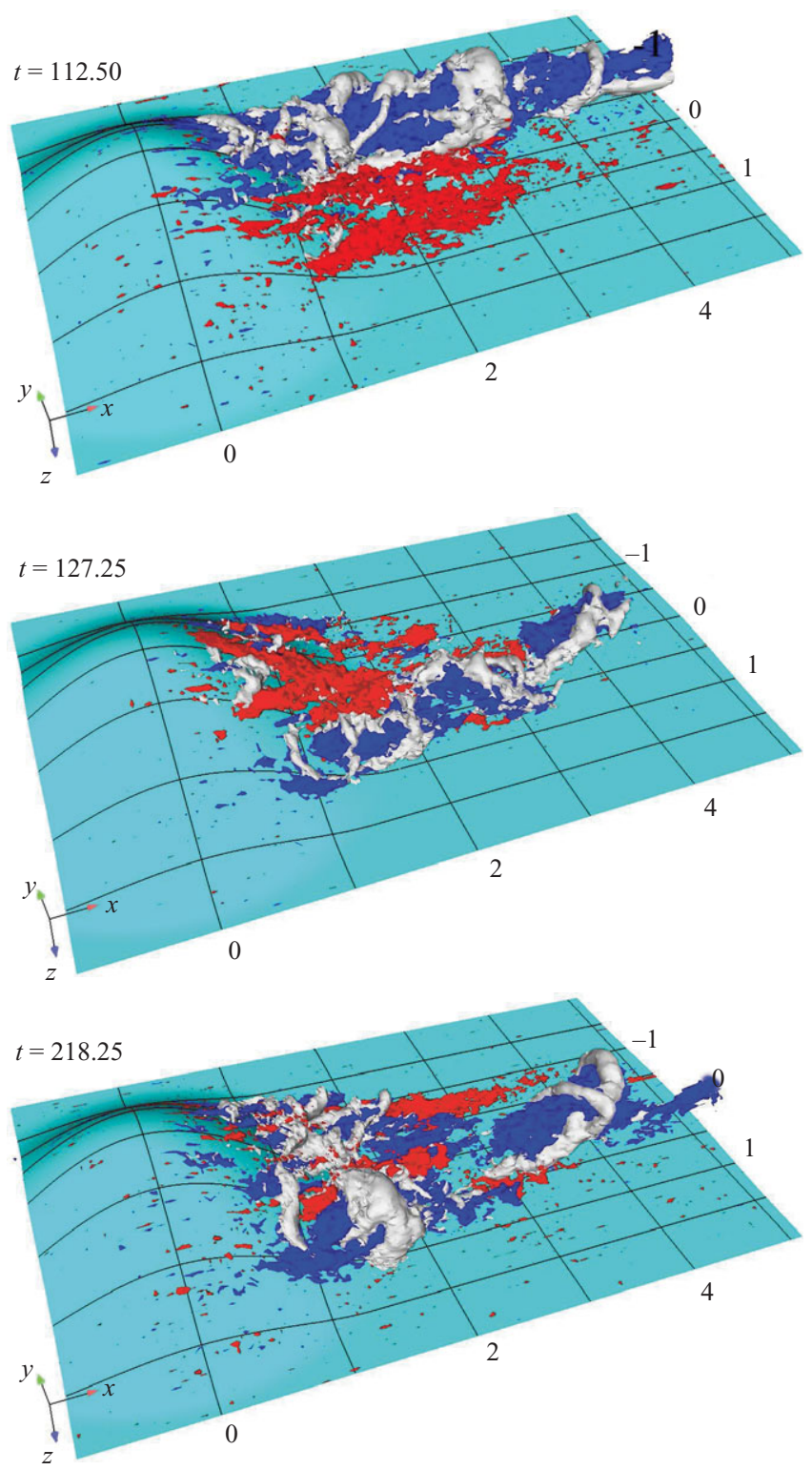

FIGURE 25. Visualization of the instantaneous flow from GVR. Blue, isosurface of streamwise velocity fluctuations $u-\langle u\rangle=-0.25$. Red, isosurface of streamwise velocity fluctuations $u-\langle u\rangle=0.25$. White, isosurface of pressure fluctuations $p-\langle p\rangle=-0.06$.

over 3000 fields to obtain converged modes. The approach taken here for elucidating structural features was to juxtapose isosurfaces of pressure with streamwise velocity fluctuations. This turns out to be instructive in so far as it brings out some interesting relationships between streamwise velocity streaks and hairpin-like vortical structures shed from the surface.

Figure 25 shows three such visualizations selected so as to bring out salient features. The plots contain three groups of isosurfaces. Two represent streamwise velocity 
fluctuations, $u-\langle u\rangle=0.25$ (red) and -0.25 (blue), and the third shows (light grey) pressure fluctuations $p-\langle p\rangle=-0.06$.

It is well known that coherent structures are associated with local minima of the pressure field (Jeong \& Hussain 1995; Dubief \& Delcayre 2000). Previous studies (Fröhlich et al. 2005; García-Villalba, Fröhlich \& Rodi 2006) have demonstrated that the field of pressure fluctuations is a more effective indicator of coherent structures than the more commonly used instantaneous pressure. This is because isosurfaces of the latter are influenced by the spatially variable average pressure field, which is unrelated to instantaneous structures. This is avoided when $\langle p\rangle$ is subtracted from the instantaneous value. It might be presumed that vorticity contours, the $\lambda_{2}$ criterion or the $Q$ criterion (Jeong \& Hussain 1995) would be preferable. However, in the present flow, the Reynolds number is relatively high, in which case intense small-scale vortical structures captured by these criteria tend to obscure the large-scale vortical structures of primary interest. Figure 25 (and corresponding animations) shows that in the wake, distinct, large-scale, elongated regions of flow moving faster than the mean flow (red) and more slowly than the mean (blue) are formed. These regions are irregular in the sense that within some periods of time only one of each type is present, while at other times, both occur together, typically one on either side of the hill. Any one of the flow conditions lasts, typically, for several time units before it is convected away or decays. There is no particular frequency to their occurrence, as shown below by reference to spectra of velocity fluctuations in this zone.

Coherent structures (light-grey) are observed to form in the leeward of the hill and are convected downstream. Many of them have the shape of a hairpin vortex, and they are rapidly deformed by the high level of turbulence around them. The presence of hairpin-shaped vortices in the flow behind a hill is not unexpected. Acarlar \& Smith (1987) generated such vortices experimentally in a laminar boundary layer obstructed by a hemisphere. Moreover, Lardeau, Tessicini \& Leschziner (2007) show, by way of highly resolved LES, that hairpin vortices arise when the present hill is subjected to a boundary layer at low Reynolds numbers. However, the pattern here is more complex because the boundary layer is fully turbulent and the Reynolds number is about 100 times larger.

It is interesting to note that the vortical structures 'ride' preferentially on the lowspeed regions. In a sense, this scenario is similar to that of vortex packets described by Adrian (2007, figure 12) in turbulent boundary layers. Here, however, the hairpin-like structures cover the whole shear-layer thickness.

\subsection{Spectra}

Time signals of the flow at several locations have been recorded in GVR's simulation over a period of about $200 \mathrm{H} / U_{\text {ref }}$. From these, spectra were determined by using a Fourier transform with a Hamming window (Oppenheim \& Schafer 1989), and segments of length 4096 (standard spectrum) and 1024 (pre-multiplied spectrum), spanning a length of 37.1 and $9.3 \mathrm{H} / U_{\text {ref }}$, respectively. The signal was decomposed into several overlapping segments over which averaging was performed. These parameters were selected so as to obtain a compromise between the smoothness of the spectrum and the width of the frequency window covered. In particular, it was necessary to reduce the length of the segments for the pre-multiplied spectra because they are represented in a linear-log form, which tends to accentuate high-frequency oscillations relative to the log-log form.

In the experiment of Ma \& Simpson (2005), time signals were recorded in the symmetry plane at $x / H=3.63$ and at various heights. Here, only the location 
(a)

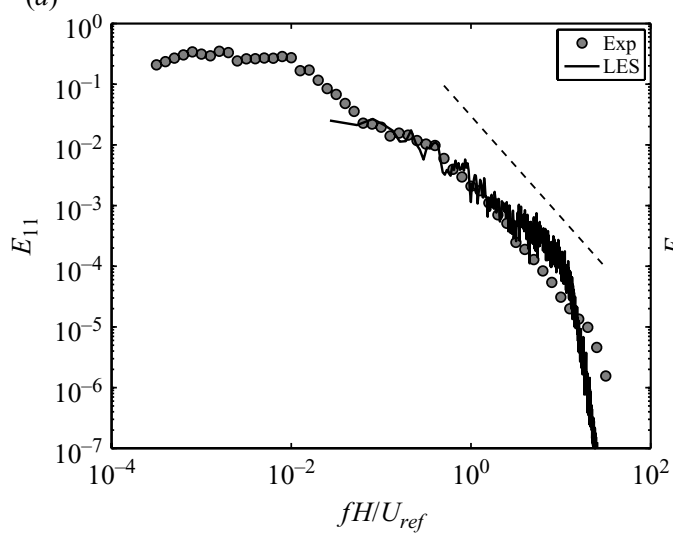

(b)

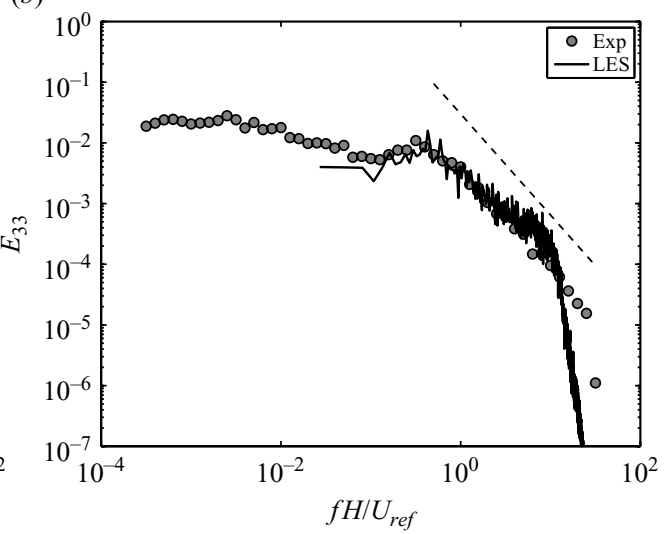

Figure 26. Comparison of power spectra of velocity fluctuations between GVR and experimental data from Ma \& Simpson (2005). $x / H=3.63, y / H=0.358, z / H=0$. (a) Streamwise velocity spectra; (b) spanwise velocity spectra. The dashed line has a $-5 / 3$ slope.

$y / H=0.36$ is compared to the simulation, because this is roughly in the middle of the boundary layer where the turbulent kinetic energy is maximum (figure 23). Agreement at other heights is similar in both qualitative and quantitative terms. Figure 26 shows the power spectrum of streamwise, $E_{11}$, and spanwise, $E_{33}$, velocity fluctuations. The frequency range in the experiment is wider because its duration, in real time, is 2-3 orders of magnitude longer than the interval covered by the simulation. At the highfrequency end of the spectrum, the decay returned by the simulation is faster than that in the experiment. For frequencies higher than $f \sim 6 U_{\text {ref }} / H$, there is some piling-up of energy, and the spectrum is slightly overpredicted by the simulation. This may be a consequence of numerical noise, which is introduced by the numerical scheme at the smallest resolved scale, thus contaminating the high-frequency end of the spectrum. Apart from this sub-range, agreement between experiment and simulation is very good over at least two decades in $f$, including the region with a $-5 / 3$ slope. It is relevant to point out that no pronounced peak is observed in the spectra, which demonstrates that the introduction of the periodic inflow with frequency $f_{\text {inf }}=0.556 U_{\text {ref }} / H(\S 3.3)$ does not have an adverse influence on the region of interest.

The power spectrum does not provide insight into how much energy is contained within a given frequency range - specifically on how much energy is contained in the very low-frequency range that is not properly represented in the LES. This deficiency can be addressed by constructing the pre-multiplied spectrum $f E_{11}\left(f E_{33}\right)$, which measures the streamwise (spanwise) kinetic energy contained in a logarithmic frequency interval centred at $f$ (del Álamo \& Jiménez 2003). The pre-multiplied spectra are displayed in figure 27. This representation clearly shows that a nonnegligible amount of energy is contained in low-frequency modes of the streamwise velocity component, but this does not apply to the spanwise component. The premultiplied spectra also show, more clearly than the power spectra, the pile-up of energy at the high-frequency end.

As noted above, the pre-multiplied spectrum derived from GVR's simulation was constructed by splitting the time signal into segments of length $9.3 \mathrm{H} / U_{\text {ref }}$ and overlapping them to enhance smoothness. Although it appears from figure 27 that the simulation does not cover the low-frequency peak at all, this is an artefact of 

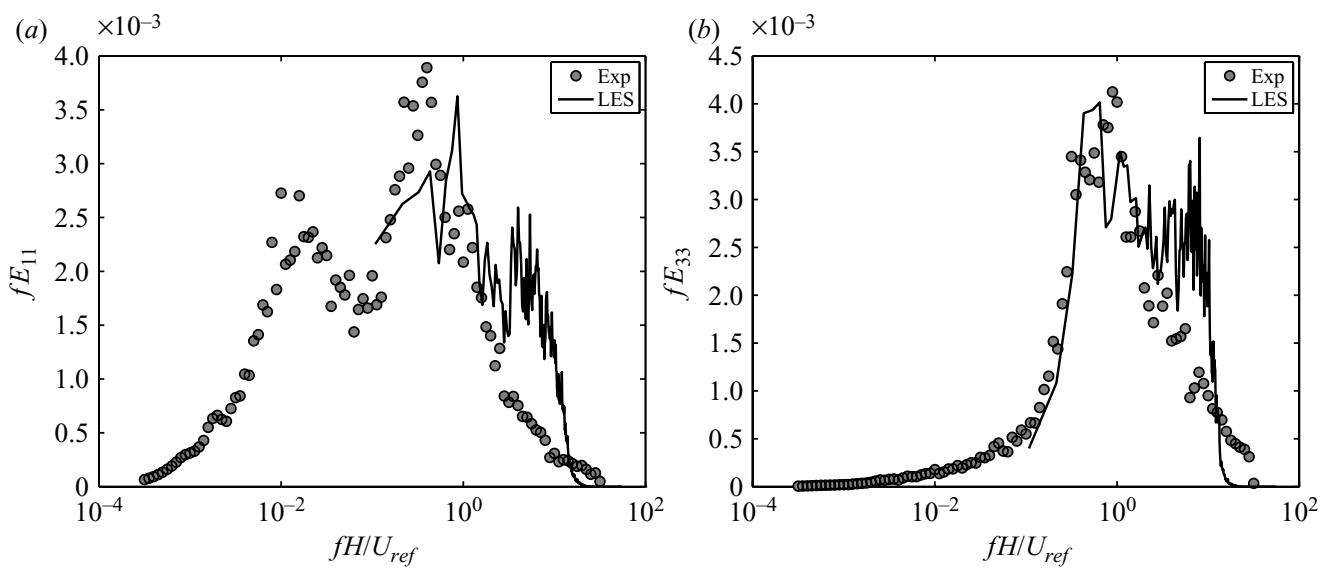

FIGURE 27. Comparison of pre-multiplied power spectra of velocity fluctuations between GVR and experimental data from Ma \& Simpson (2005). $x / H=3.63, y / H=0.358, z / H=0$. (a) Streamwise velocity spectra; (b) spanwise velocity spectra.

the splitting of the signal. Some of the motions at this frequency range are in fact contained in the simulation. These motions are associated with the elongated regions of alternating low and high speed which were discussed in relation to figure 25 . To obtain a smoother spectrum at the low-frequency end, the simulations have to be run over an extremely long period of time, exceeding the actual duration by perhaps two orders of magnitude.

\section{Conclusions}

The results of two entirely independent large-eddy simulations of the flow over a three-dimensional hill have been reported, analysed and compared to experimental data. The study was motivated by the importance attached to this flow by the turbulence modelling and simulation community, as a generic test case that presents challenging features encountered in a broad range of practical three-dimensional flows separating from curved surfaces.

The mean flow in the leeward of the hill is characterized by a very thin, but influential, separated region, which is very difficult to resolve accurately in the simulation (as it appears to be in corresponding experiments). Both simulations show that the upstream portion of the reverse-flow layer tapers very gently towards the separation line, and this is not resolved by the experiment. As a consequence, the separation line is predicted to lie well upstream of that deduced from the measurement. The present simulations show that it is essential to have an extremely fine resolution in the wall-normal direction very close to the wall in this region. It appears that LL's simulation satisfies this particular requirement, while GVR's wall-normal resolution in the separated zone may not be quite fine enough. It has been shown that the pressure distribution is a very sensitive indicator of the structure of the separation zone, even though it is very thin. This structure is very complex and has been studied in detail by reference to the stream lines constructed with the velocity components that lie within wall-parallel surfaces at various wall-normal distances. At the wall, on either side of the hill centre plane, a large focal point is present on the outer part of the hill surface, from which two counter-rotating vortices emanate and evolve into the hill wake. Somewhat farther away from the wall, two additional vortices with a 
sense of rotation opposite to that of the outer ones develop close to the centre plane. The latter originate from the separation node that is at the apex of the separation line on the centre plane. Reattachment occurs roughly at the leeward foot of the hill, and both simulations return virtually the same reattachment location.

The wake is characterized by a combination of streamwise acceleration and transverse circulation. The former is associated with the wake-recovery process and the latter with secondary motions originating from a combination of the streamwise realignment of the vorticity in the upstream boundary layer and substantial additional vorticity generated at the hill surface. The latter is an amalgam of vorticity components of opposite sense originating from the outer focal points and from the separation node at the apex of the separation line. As the wake evolves, the vortices shed from the outer focal points are overwhelmed, through a diffusive mechanism, by the vorticity shed from the separation node. As a result, the latter vortices dictate the sense of rotation of the secondary motion in the wake. The influence of wall-normal resolution in the wake, relative to streamwise and spanwise resolution, is weaker than in the separated region, and in this area GVR's grid is distinctly superior to LL's grid, thus yielding a more faithful representation of the wake structure.

The main characteristics of the flow are well reproduced by both simulations, and there is a gratifying level of agreement between them in respect of the dimensions of the separation zone, the mean velocity, the Reynolds stresses, the principal topological features and the wake structure - the last reproduced especially well by GVR's simulation. There are, however, also some non-negligible differences between them, as well as between both simulations and the experiment. The most important difference in the latter category concerns the topological features observed at the wall. In the experiment (Byun \& Simpson 2006), two foci and one saddle point were observed in the separation region. The region of reattachment could not be measured, and Byun \& Simpson conjectured that, to fulfil the topological rules, the reattachment region should contain one reattachment node and two additional saddle points (i.e. three nodes and three saddle points, in total). Both simulations return a different topology, featuring four nodes and four saddle points. Furthermore, it has been shown that this is consistent with the predicted development of the mean streamwise vorticity in the wake, which is in good agreement with the HWA measurements of Ma \& Simpson (2005). This observation supports the validity of the topology predicted by the simulations. A second difference relative to the experiments concerns the prediction of the location of the separation line. The origin of the unexpected experimental observation of a patch of high turbulence energy well upstream of the experimentally deduced separation region has been elucidated by the simulations. These demonstrate that the area of high turbulence energy is a consequence of the highly unsteady separation process, resulting in a very thin time-mean reverse-flow layer extending well upstream of the location determined experimentally. Hence, the patch of high turbulence energy, shown to be a consequence of high turbulence production, is closely associated with the separation process.

As regards differences between the two simulations, the most important relates to the wall pressure in the separation region. A close examination of the flow in the separated region has revealed that these differences originate from the extreme sensitivity of the pressure gradient to the variation of the wall-normal thickness of the reverse-flow layer. This sensitivity highlights the extreme resolution challenges posed by three-dimensional separation from curved surfaces.

Although the study has targeted primarily the resolution of the mean-flow characteristics, some useful observations have also been made in respect of unsteady 
features and processes. It has been shown that, while the separation region is highly unsteady, the flow is separated at all times at least over some parts of the surface. Based on the measurement of bimodal velocity PDF's, Byun (2005) speculated on the possibility that the separation is intermittent, involving a switching between detached and attached flow. This scenario is not supported by the simulations. Another interesting feature observed in the simulations is the presence in the wake of alternating regions of fluid in which the streamwise velocity is higher or lower than the mean. Associated with the low-speed regions, coherent structures have been identified, some having the form of hairpin vortices. These events are very energetic, as is evident in time signals of the velocity recorded in the wake.

Apart from shedding light on important fundamental aspects of separation from curved surfaces, the present results, alongside the experimental data, may be claimed to provide a reliable benchmark for assessing the capabilities of other computational techniques, in particular hybrid LES-RANS approaches, thereby contributing to progress in obtaining reliable calculation methods for three-dimensional separating flows in practice.

García-Villalba acknowledges the financial support provided by the German Research Foundation (DFG) and by the European Science Foundation through the COST Action P20. The GVR simulation was performed on the national super computer NEC SX-8 at the High Performance Computing Center Stuttgart (HLRS). The Karlsruhe team would like to thank Dr. J. G. Wissink for his help with the grid generation. Leschziner and $\mathrm{Li}$ are grateful to the UK Engineering and Physical Sciences Research Council (EPSRC) and BAe Systems for supporting the research financially as well as through the provision of supercomputer resources on the national facility HPCx at the Daresbury Laboratory, UK. The authors are grateful to Prof. R. L. Simpson for helpful information provided on the experimental studies and to Dr. R. Ma and Dr. G. Byun for providing the experimental data in electronic format.

\section{REFERENCES}

ACARlaR, M. S. \& SMith, C. R. 1987 A study of hairpin vortices in a laminar boundary layer. Part 1. Hairpin vortices generated by a hemisphere protuberance. J. Fluid Mech. 175, 1-41.

AdRian, R. J. 2007 Hairpin vortex organization in wall turbulence. Phys. Fluids 19, 041301.

DEl Álamo, J. C. \& JimÉnez, J. 2003 Spectra of the very large anisotropic scales in turbulent channels. Phys. Fluids 15, L41-L44.

ApsLEY, D. D. \& CASTRO, I. P. 1997 Flow and dispersion over hills: comparison between numerical predictions and experimental data. J. Wind Engng Ind. Aerodyn. 67-68, 375-386.

Aubertine, C. D. \& Eaton, J. K. 2005 Turbulence development in a non-equilibrium turbulent boundary layer with mild adverse pressure gradient. J. Fluid Mech. 532, 345-364.

Benhamadouche, S., Uribe, J., Jarrin, N. \& Laurence, D. 2005 Large eddy simulation of a symmetric bump on structured and unstructured grids, comparisons with RANS and T-RANS models. In Proceedings of the Fourth Intl Sympos. on Turbulence and Shear Flow Phenomena. Williamsburg, Virginia, pp. 325-330.

BLAKE, W. K. 1975 A statistical description of pressure and velocity fields at the trailing edge of a flat strut. David Taylor Naval Ship R\&D Center Report 4241, Bethesda, Maryland.

BReuer, M. \& RoDi, W. 1996 Large eddy simulation of complex turbulent flows of practical interest. In Flow simulation with high performance computers II (ed. E. H. Hirschel), Notes on Numerical Fluid Mechanics, vol. 52, pp. 258-274. Vieweg.

BYun, G. 2005 Structure of three-dimensional separated flow on symmetric bumps. PhD thesis, Virginia Polytechnic Institute and State University, Blacksburg.

BYun, G. \& Simpson, R. L. 2006 Structure of three-dimensional separated flow on an axisymmetric bump. AIAA J. 44 (5), 999-1008 (also AIAA Paper 2005-0113). 
Byun, G., Simpson, R. L. \& Long, C. H. 2004 Study of vortical separation from three-dimensional symmetric bumps. AIAA J. 42 (4), 754-765.

DAvidson, L. \& DAhlströHM, S. 2005 Hybrid LES-RANS: computation of the flow around a three-dimensional hill. In Engng Turbulence Modelling and Experiments 6 (ed. W. Rodi \& M. Mulas). Elsevier.

Ding, L. \& Street, R. L. 2003 Numerical study of the wake structure behind a three-dimensional hill. J. Atmos. Sci. 60, 1678-1690.

Dubief, Y. \& DelcaYre, F. 2000 On coherent-vortex identification in turbulence. J. Turbul. 1.

Fröhlich, J., Mellen, C. P., Rodi, W., Temmerman, L. \& Leschziner, M. A. 2005 Highly resolved large-eddy simulation of separated flow in a channel with streamwise periodic constrictions. J. Fluid Mech. 526, 19-66.

Fureby, C., Alin, N., Wikström, N., Menon, S., Svanstedt, N. \& Persson, L. 2004 Large-eddy simulation of high-Reynolds number wall-bounded flows. AIAA J. 42 (3), 457-468.

García-Villalba, M., FröHlich, J. \& Rodi, W. 2006 Identification and analysis of coherent structures in the near field of a turbulent unconfined annular swirling jet using large eddy simulation. Phys. Fluids 18, 055103.

García-Villalba, M., Stoesser, T., von Terzi, D., Wissink, J. G., Fröhlich, J. \& Rodi, W. 2007 Large eddy simulation of turbulent separated flow over a three-dimensional hill. In Advances in Turbulence XI (ed. J. M. L. M. Palma \& A. Silva Lopes), pp. 627-629.

Germano, M., Piomelli, U., Moin, P. \& Cавот, W. H. 1991 A dynamic subgrid-scale eddy viscosity model. Phys. Fluids 3, 1760-1765.

Hinterberger, C. 2004 Dreidimensionale und tiefengemittelte large-eddy-simulation von Flachwasserströmungen. PhD thesis, University of Karlsruhe.

Hunt, J. C. R., Abell, C. J., Peterka, J. A. \& Woo, H. 1978 Kinematical studies of the flow around free or surface-mounted obstacles: applying topology to flow visualization. J. Fluid Mech. 86, 179-200.

Hunt, J. C. R. \& SNyder, W. H. 1980 Experiments on stably and neutrally stratified flow over a model three-dimensional hill. J. Fluid Mech. 96, 671-704.

Jeong, J. \& Hussain, F. 1995 On the identification of a vortex. J. Fluid Mech. 285, 69-94.

KrAJNović, S. 2008 Large eddy simulation of the flow around a three-dimensional axisymmetric hill. Flow Turbul. Combust. 81 (1-2), 189-204.

Lardat, R. \& Leschziner, M. A. 1998 A Navier-Stokes solver for LES on parallel computers. Tech Rep. University of Manchester.

Lardeau, S., Tessicini, F. \& Leschziner, M. A. 2007 Identification of hairpin-type flow structures in separated flow behind a three-dimensional hill using POD. In Proceedings of the Fifth Intl Sympos. on Turbulence and Shear Flow Phenomena. Munich.

LE, H. \& MoIn, P. 1991 An improvement of fractional step methods for the imcompressible Navier-Stokes equations. J. Comput. Phys. 92, 369-379.

Li, N. \& LesChZiner, M. A. 2007 Highly resolved simulation of flow over a three-dimensional hill. In Advances in Turbulence XI (ed. J. M. L. M. Palma \& A. Silva Lopes), pp. 407-409.

Lilly, D. K. 1992 A proposed modification of the Germano subgrid-scale closure method. Phys. Fluids 4, 633-635.

MA, R. \& Simpson, R. L. 2005 Characterization of turbulent flow downstream of a three-dimensional axisymmetric bump. In Proceedings of the Fourth Intl Sympos. on Turbulence and Shear Flow Phenomena. Williamsburg, Virginia, pp. 1171-1176.

ManhaRT, M. 1998 Vortex shedding from a hemisphere in a turbulent boundary layer. Theor. Comput. Fluid Dyn. 12, 1-28.

Oppenheim, A. V. \& Schafer, R. W. 1989 Discrete-Time Signal Processing. Prentice Hall.

Patel, N. \& Menon, S. 2007 Structure of flow separation and reattachment behind an axisymmetric hill. J. Turbul. 8 (36), 1-24.

Patel, N., Stone, C. \& Menon, S. 2003 Large-eddy simulation of turbulent flow over an axisymmetric hill. AIAA Paper 2003-0967.

Persson, T., Liefvendahl, M., Benson, R. E. \& Fureby, C. 2006 Numerical investigation of the flow over an axisymmetric hill using LES, DES and RANS. J. Turbul. 7 (4), 1-17.

PierCE, C. D. 2001 Progress-variable approach for large-eddy simulation of turbulent combustion. $\mathrm{PhD}$ thesis, Stanford University. 
Rhie, C. M. \& Chow, W. L. 1983 Numerical study of the turbulent flow past an airfoil with trailing edge separation. AIAA J. 21 (11), 1061-1068.

Simpson, R. L., Long, C. H. \& ByUN, G. 2002 Study of vortical separation from an axisymmetric hill. Intl J. Heat Fluid Flow 23 (5), 582-591.

Song, S., DeGraAfF, D. B. \& Eaton, J. K. 2000 Experimental study of a separating, reattaching, and redeveloping flow over a smoothly contoured ramp. Intl J. Heat Fluid Flow 21 (5), 512-519.

StONe, H. L. 1968 Iterative solution of implicit approximations of multidimensional partial differential equations for finite difference methods. SIAM J. Numer. Anal. 5, 530-558.

Tessicini, F., Li, N. \& Leschziner, M. A. 2007 Large-eddy simulation of three-dimensional flow around a hill-shaped obstruction with a zonal near-wall approximation. Intl J. Heat Fluid Flow 28 (5), 894-908.

Visbal, M. R., Rizzetta, D. P. \& Mathew, J. 2007 Large-eddy simulation of turbulent flow past a 3-d bump. AIAA Paper 2007-917.

WANG, C., JANG, Y. J. \& Leschziner, M. A. 2004 Modelling two- and three-dimensional separation from curved surfaces with anisotropy-resolving turbulence closures. Intl J. Heat Fluid Flow 25 (3), 499-512.

Wang, M. \& MoIn, P. 2002 Dynamic wall modelling for large-eddy simulation of complex turbulent flows. Phys. Fluids 14 (7), 2043-2051.

Wasistho, B. \& Squires, K. D. 2001 Numerical investigation of the separated flow over a smoothly contoured ramp. In Proceedings of the Second Intl Sympos. on Turbulence and Shear Flow Phenomena.

Werner, H. \& Wengle, H. 1993 Large-eddy simulation of turbulent flow over and around a cube in a plate channel. In Eighth Sympos. on Turbulent Shear Flows, pp. 155-168. Springer.

Wissink, J. G., Michelassi, V. \& RoDi, W. 2004 Heat transfer in a laminar separation bubble affected by oscillating external flow. Intl J. Heat Fluid Flow 25, 729-740. 